\title{
Filgotinib suppresses HIV-1-driven gene transcription by inhibiting HIV-1 splicing and T cell activation
}

\author{
Yang-Hui Jimmy Yeh, ${ }^{1}$ Katharine M. Jenike, ${ }^{2}$ Rachela M. Calvi, ${ }^{3}$ Jennifer Chiarella, ${ }^{3}$ Rebecca Hoh, ${ }^{4}$ Steven G. Deeks, ${ }^{4}$ and Ya-Chi Ho ${ }^{1}$ \\ 'Department of Microbial Pathogenesis, Yale University School of Medicine, New Haven, Connecticut, USA. ²Human Cenetics PhD Program, Johns Hopkins University School of Medicine, Baltimore, \\ Maryland, USA. ${ }^{3}$ Department of Neurology, Yale University School of Medicine, New Haven, Connecticut, USA. ${ }^{4}$ Department of Medicine, UCSF, San Francisco, California, USA.
}

\begin{abstract}
Despite effective antiretroviral therapy, HIV-1-infected cells continue to produce viral antigens and induce chronic immune exhaustion. We propose to identify HIV-1-suppressing agents that can inhibit HIV-1 reactivation and reduce HIV-1-induced immune activation. Using a newly developed dual-reporter system and a high-throughput drug screen, we identified FDAapproved drugs that can suppress HIV-1 reactivation in both cell line models and CD4+ T cells from virally suppressed HIV-1infected individuals. We identified 11 cellular pathways required for HIV-1 reactivation as druggable targets. Using differential expression analysis, gene set enrichment analysis, and exon-intron landscape analysis, we examined the impact of drug treatment on the cellular environment at a genome-wide level. We identified what we believe to be a new function of a JAK inhibitor, filgotinib, that suppresses HIV-1 splicing. First, filgotinib preferentially suppresses spliced HIV-1 RNA transcription. Second, filgotinib suppresses HIV-1-driven aberrant cancer-related gene expression at the integration site. Third, we found that filgotinib suppresses HIV-1 transcription by inhibiting T cell activation and by modulating RNA splicing. Finally, we found that filgotinib treatment reduces the proliferation of HIV-1-infected cells. Overall, the combination of a drug screen and transcriptome analysis provides systematic understanding of cellular targets required for HIV-1 reactivation and drug candidates that may reduce HIV-1-related immune activation.
\end{abstract}

\section{Introduction}

Despite effective antiretroviral therapy (ART), HIV-1 persists in the latent reservoir, which is a major barrier to finding a cure (1-3). Treatment interruptions lead to viral rebound from latently infected cells (4). One of the strategies to cure HIV-1 infection is the shockand-kill strategy, which reverses HIV-1 latency and exposes HIV-1infected cells for immune clearance $(5,6)$. To prevent systemic toxicity, an ideal latency-reversing agent should reverse HIV-1 latency without causing global $\mathrm{T}$ cell activation. Extensive small-molecule compound library screens have identified latency-reversing agents, such as histone deacetylase inhibitors, protein kinase $\mathrm{C}$ agonists, and noncanonical NF- $\mathrm{KB}$ activators, that can reactivate HIV-1 in vivo (5, 7-10). Yet, while latency-reversing agents can induce HIV-1 RNA transcription, recent evidence suggests that latency reversal without $\mathrm{T}$ cell activation is not sufficient to induce antigen presentation and immune clearance $(11,12)$. Understanding the cellular environment supporting HIV-1 transcription is required to develop effective HIV-1 eradication strategies.

Latent HIV-1 proviruses in quiescent memory $\mathrm{CD}^{+} \mathrm{T}$ cells are largely transcriptionally inactive. This is because of the lack of active transcription factors and the Tat positive-feedback loop (13-17), transcriptional blocks (18), and the repressive chromatin environment (19-22). Upon antigen stimulation, $\mathrm{T}$ cell receptor

Conflict of interest: $\mathrm{YCH}$ receives research grants from Gilead Sciences awarded to Yale University.

Copyright: (5) 2020, American Society for Clinical Investigation.

Submitted: February 19, 2020; Accepted: June 10, 2020; Published: August 17, 2020

Reference information: J Clin Invest. 2020;130(9):4969-4984.

https://doi.org/10.1172/JCl137371. signaling leads to AP-1 (23), NFAT (24), and NF- $\kappa$ B (25) transcription factor activation, nuclear translocation, and binding to the HIV-1 promoter, and induces robust HIV-1 reactivation. Even without latency-reversing agents, stochastic antigen stimulations in vivo reactivate HIV-1 from latency at about 0.4 events per day $(26,27)$. This is evidenced by the fact that HIV-1 RNA expression can be readily detected in $\mathrm{CD} 4^{+} \mathrm{T}$ cells from ART-treated, virally suppressed HIV-1-infected individuals in the absence of ex vivo latency reversal $(28,29)$. Therefore, despite effective ART, chronic HIV-1 antigen production leads to chronic immune activation (28, 30-33), immune exhaustion (34), the residual immune dysregulatory syndrome $(35,36)$, premature atherosclerosis $(37,38)$, and accelerated aging $(39,40)$. Until a safe, scalable, affordable, and generalizable HIV-1 eradication strategy is available, HIV-1infected individuals will continue to suffer from chronic immune activation (41). To halt HIV-induced immune dysfunction and chronic immune activation, therapeutic strategies that can inhibit HIV-1 transcription are required.

ART inhibits viral enzyme function or viral entry but does not inhibit HIV-1 transcription and viral antigen production. While immunomodulatory strategies can enhance immune effector functions, stopping antigen production from HIV-1-infected cells is the key to blocking HIV-1-induced immune dysfunction. To target HIV-1 transcription effectively, a comprehensive understanding of cellular pathways both sufficient and necessary for HIV-1 transcription is needed. HIV-1 latency-reversing agent searches such as drug screens $(5,42)$ and cellular factor screens $(43,44)$ have identified drugs or cellular pathways sufficient for HIV-1 reactivation, but not necessarily those required for HIV-1 transcription. Drug and cellular factor screens searching for agents 
that can suppress HIV-1 transcription would identify cellular pathways required for HIV-1 transcription, such as Tat transactivation, mTOR signaling, cation transport, and estrogen receptor signaling (45-49). However, while these HIV-1-suppressing agents were reported individually, a full picture of targetable cellular pathways necessary for HIV-1 transcription remains unclear.

Here we combined a newly developed dual-reporter highthroughput drug screen system and 3 genome-wide transcriptome analysis approaches to systematically identify drugs and cellular pathways that can inhibit HIV-1 transcription after the establishment of latency. We confirmed that the androgen antagonist and modest diuretic spironolactone (50); a GTP-depleting agent, mycophenolic acid (51); and a JAK inhibitor, ruxolitinib (52), can suppress HIV-1 transcription as previously reported. Importantly, we identified a JAK inhibitor, filgotinib, that can inhibit HIV-1driven aberrant host gene expression in addition to inhibiting HIV-1 transcription. Filgotinib is an immunomodulatory agent widely used in autoimmune diseases such as rheumatoid arthritis (53), ankylosing spondylitis (54), psoriatic arthritis (55), and Crohn's disease (56). In this study, our goal was both to probe for cellular pathways required for HIV-1 transcription and to identify drugs that can suppress HIV-1 transcription.

\section{Results}

A dual-reporter screen identified FDA-approved drugs that can preferentially inhibit HIV-1 transcription as HIV-1-suppressing agents. To identify drugs that can preferentially inhibit HIV-1 expression, we developed a dual-reporter HIV-1-infected Jurkat T cell system. These dual-reporter HIV-1-infected Jurkat T cell clones harbor both HIV-1-green fluorescent protein (HIV-1-dsGFP) reporter (42) and $\mathrm{EF} 1 \alpha$-driven blue fluorescent protein (EF1 $\alpha$-dsBFP) lentiviral reporter (Supplemental Figure 1A; supplemental material available online with this article; https://doi.org/10.1172/JCI137371DS1). HIV-1-dsGFP reports HIV-1 expression levels, while EF1 $\alpha$-dsBFP serves as a counterscreen to measure drug effects on host gene transcription. Decreased HIV-1-dsGFP expression with minimal changes in EF1 $\alpha$-dsBFP expression indicates preferential suppression of HIV-1 expression over host gene expression. Both dsGFP and dsBFP are destabilized (ds) through PEST sequence-mediated ubiquitination, leading to a short half-life of 2 hours (42) to reflect HIV-1-dsGFP and EF1 $\alpha$-dsBFP expression levels in real time. We targeted HIV-1-dsGFP to the endoplasmic reticulum through the signal peptide and targeted EF1 $\alpha$-dsBFP to the nucleus through a nuclear localization signal to prevent fluorescence resonance energy transfer between BFP and GFP. Different from J-Lat T cell lines, which have low or no HIV-1 transcription at baseline, we identified clones that have high basal levels $(>40 \%)$ of HIV-1 transcription, namely 1B6-du, 5F9-du, and 6C6-du. This strategy provides a wide dynamic range of HIV-1-dsGFP expression and allows us to identify drugs that can suppress HIV-1 expression. Further, we identified clones in which HIV-1 reporters were integrated into introns of actively transcribed genes to recapitulate HIV-1 integration observed in HIV-1-infected individuals $(57,58)$. Overall, our dual-reporter HIV-1-infected Jurkat T cell system allows real-time detection of HIV-1 transcription states, a counterscreen reflecting cellular gene transcription state, and integration into introns recapitulating HIV-1 integration patterns observed in vivo.
The landscape of cellular pathways required for HIV-1 transcription as druggable therapeutic targets. We used an FDA-approved small-molecule compound library to identify HIV-1-suppressing agents. While this library does not contain epigenetic silencing agents, the use of FDA-approved drugs identifies agents with known clinical toxicity profiles and allows for efficient clinical application. Drugs that suppress HIV-1-dsGFP expression below 3 standard deviations from the mean with EF1 $\alpha$-dsBFP expression in the 1B6-du clone were defined as candidate HIV-1-suppressing agents (Supplemental Figure 1B and Figure 1A). From 1,430 FDA-approved small-molecule drugs, we identified 11 cellular pathways and 16 putative HIV-1-suppressing agents (Supplemental Table 1, Figure 1A, and Figure 2) that inhibit HIV-1 transcription. Our screen confirmed previously reported agents that can inhibit HIV-1 transcription, such as the JAK inhibitor ruxolitinib (59), the DNA helicase inhibitor spironolactone (50), the GTP synthesis inhibitor mycophenolic acid (51), the transcription inhibitor flavopiridol (60), and the cation transporter inhibitors levosimendan (45) and digoxin (46). Consistent with previous findings (61), mTOR inhibitors do not significantly suppress HIV-1 transcription in our model. We also identified FDA-approved drugs that can target pathways known to affect HIV-1 transcription, such as uprosertib for Akt inhibition (62) and KPT-330 for CRM1-mediated nuclear RNA export (63). Importantly, we identified drugs and cellular pathways that were not previously known to affect HIV-1 transcription, such as dovitinib, pazopanib, and ponatinib, which inhibit receptor tyrosine kinase pathways, and mitomycin C, irinotecan, and mitoxantrone, which inhibit DNA unwinding (Supplemental Table 1 and Figure 2).

Because one cell line model may not recapitulate the heterogeneous HIV-1 integration sites in vivo, we tested the effect of these 16 HIV-1-suppressing agents in 2 additional cell line clones and in $\mathrm{CD}^{+}{ }^{+} \mathrm{T}$ cells from virally suppressed HIV-1-infected individuals (Supplemental Table 2). We first examined dose-response curves and cellular viability using flow cytometry in the 2 additional cell line clones 5F9-du and 6C6-du (Supplemental Figure 1C and Supplemental Figure 2). These cell line clones harbor HIV-1dsGFP proviral reporters integrated into introns of different host genes (Supplemental Figure 2). We found that 8 drugs (filgotinib, digoxin, levosimendan, zinc pyrithione, irinotecan, mitomycin C, mycophenolic acid, and spironolactone) suppressed HIV-1-dsGFP expression without affecting EF1 $\alpha$-dsBFP expression and cellular viability in both of the additional cell lines (Supplemental Figure 2).

The JAK1 inhibitor filgotinib is an HIV-1-suppressing agent. Considering the feasibility of systemic dosing and clinical adverse effects, we focused on filgotinib, a new-generation JAK1 inhibitor that has not been reported to affect HIV-1 transcription. We used ruxolitinib, a JAK inhibitor known to suppress HIV-1 transcription (52) (ClinicalTrials.gov NCT02475655), to compare whether filgotinib differs from other JAK inhibitors in terms of its mediation of HIV-1 suppression. To examine whether filgotinib suppresses HIV-1 transcription through distinct mechanisms, we used 2 drugs known to suppress HIV-1 transcription, the DNA helicase inhibitor spironolactone (50) and the IMPDH inhibitor mycophenolic acid $(51,64)$ (NCT03262441), as positive controls. These 4 drugs (filgotinib, ruxolitinib, spironolactone, and mycophenolic acid) have minimal suppression of EF1 $\alpha$-dsBFP expression $(<0.5$ standard 
A 1,430 FDA-approved drug screen

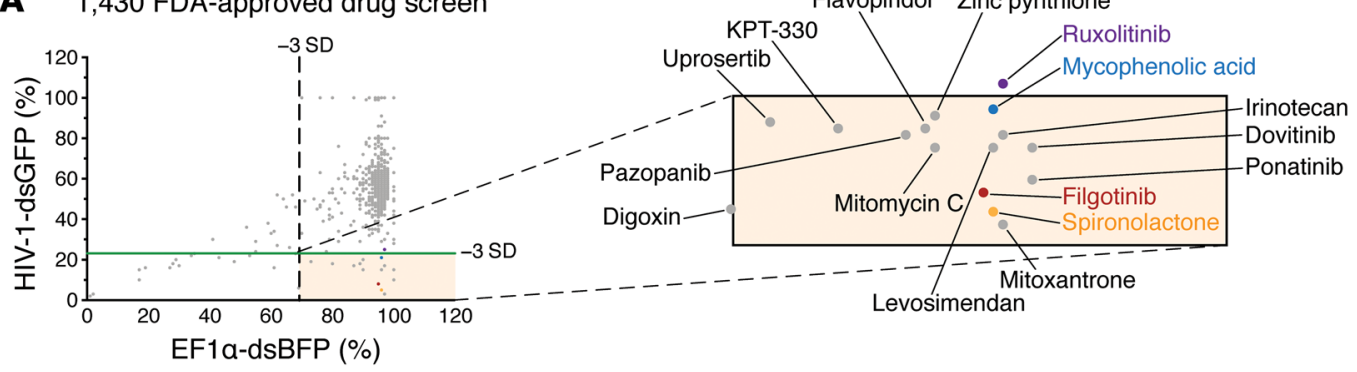

B

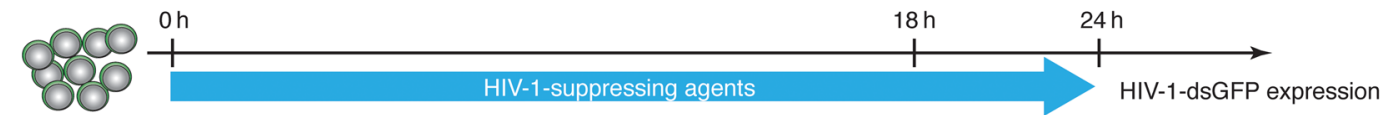

HIV-1-dsGFP Jurkat

$T$ cell clones
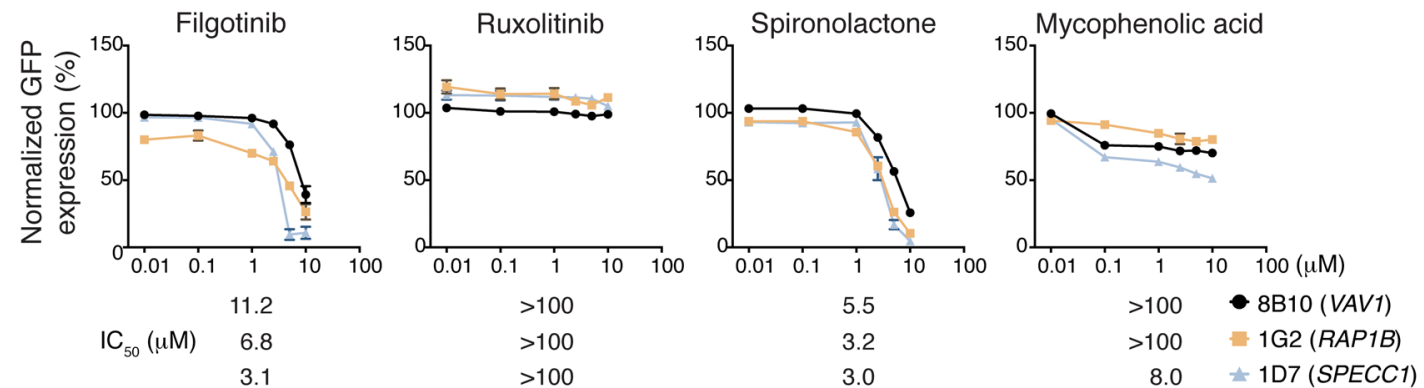

C

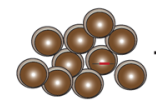

Oh

$>100$

3.0

$8.0+1 \mathrm{D} 7$ (SPECC1)

Resting CD4 ${ }^{+} \mathrm{T}$ cells from HIV-1-infected individuals
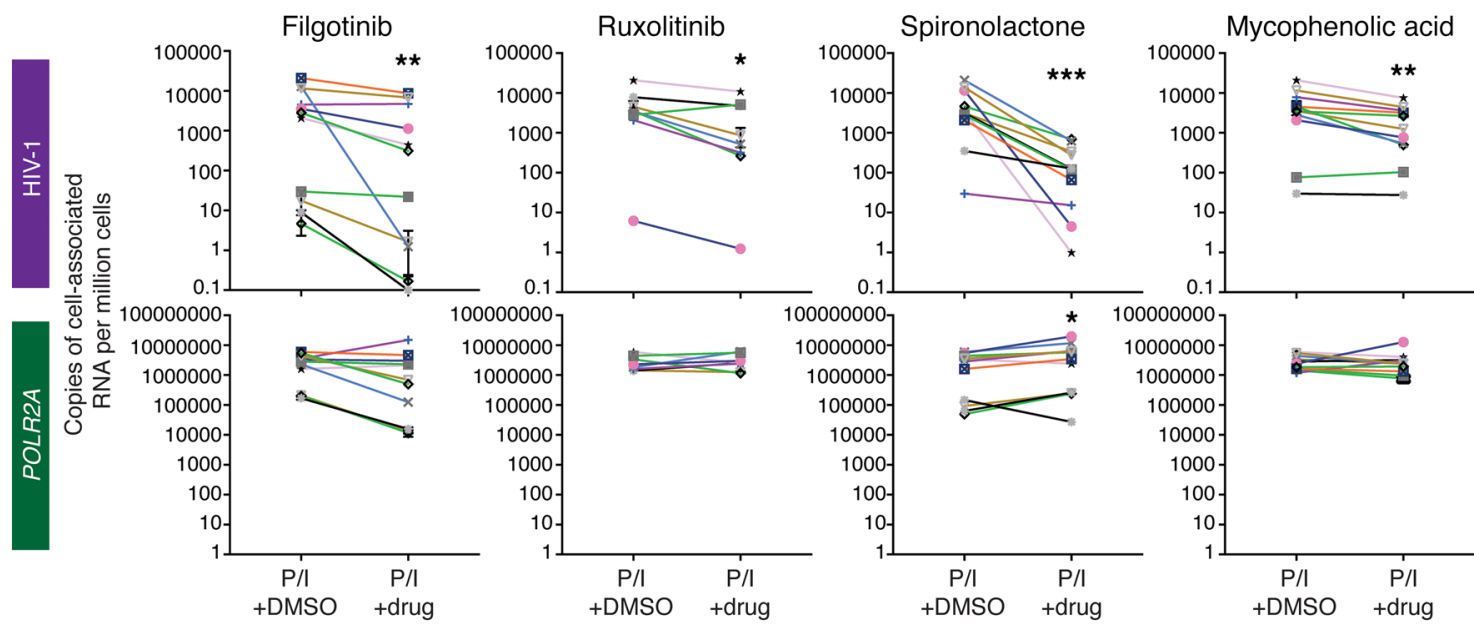

Figure 1. A dual-reporter screen identified HIV-1-suppressing agents. (A) Screening of 1,430 small-molecule compounds from an FDA-approved drug library identified 16 HIV-1-suppressing agents in dual-color Jurkat clone 1B6-du. (B) Dose-response curves of candidate HIV-1-suppressing agents in 3 cell line models. HIV-1-dsGFP expression levels were normalized to the levels in DMSO-treated samples. Error bars represent SEM from quadruplicates. (C) Cell-associated RNA levels of polyadenylated HIV-1 and a housekeeping gene, POLR2A, in CD4+ $\mathrm{T}$ cells from virally suppressed HIV-1-infected individuals upon treatment with HIV-1-suppressing agents ( $10 \mu \mathrm{M}$ for 24 hours) and PMA/ionomycin (P/I) challenge (for 6 hours) in the presence of ART ( $\mu \mathrm{M}$ tenofovir and $10 \mu \mathrm{M}$ enfuvirtide). Each color represents samples from an HIV-1-infected individual. ds, destabilized protein through PEST sequence-mediated ubiquitination, giving the fluorescent proteins a half-life of 2 hours (42) for real-time reflection of HIV-1-dsGFP and EF1 $\alpha$-dsBFP expression levels.

${ }^{*} P<0.05,{ }^{* *} P<0.01,{ }^{* * *} P<0.001$ by 2 -tailed Wilcoxon rank-sum test.

deviation from mean) and minimal cytotoxicity $(>80 \%$ cellular viability) (Supplemental Figure 2).

We first examined the dose-response curves using 3 additional cell line clones, 8B10 (harboring HIV-1-dsGFP integrated into
VAV1), 1G2 (harboring HIV-1-dsGFP integrated into RAP1B), and 1D7 (harboring HIV-1-dsGFP integrated into SPECC1) (Figure 1B). Filgotinib and spironolactone remarkably suppressed HIV-1dsGFP expression (0.6 and $0.8 \log$ reduction, respectively). The 


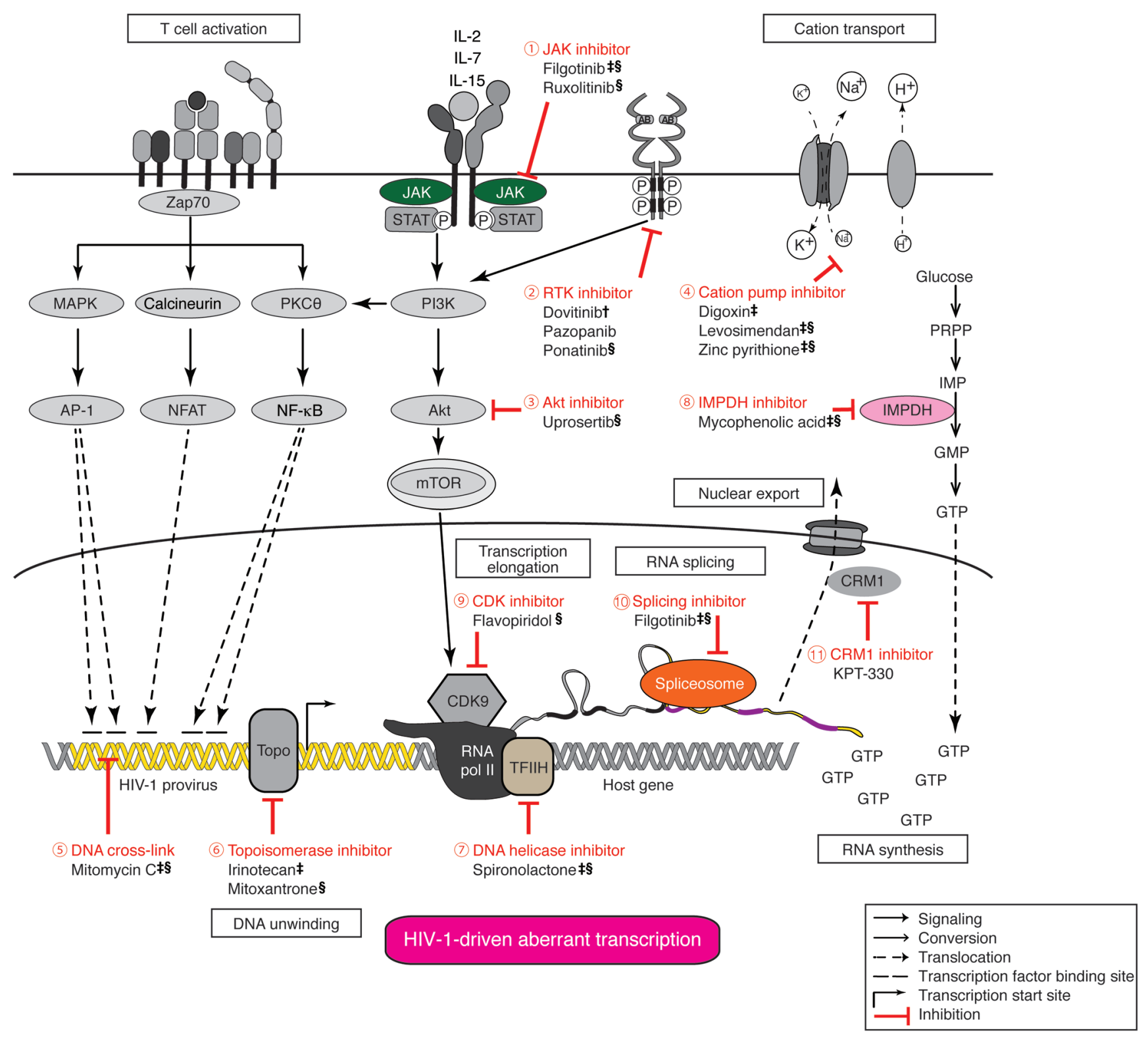

Figure 2. Therapeutic targets of HIV-1 reactivation. A high-throughput drug screen identified 11 cellular pathways critical for HIV-1 transcription after HIV-1 integration. ${ }^{\dagger}$ Preferential HIV-1 suppression in 1 additional cell line. ${ }^{\sharp} \mathrm{HIV}-1$ suppression in 2 additional cell lines. ${ }^{8} \mathrm{HIV}-1$ suppression in CD4 ${ }^{+} \mathrm{T}$ cells from virally suppressed HIV-1-infected individuals.

$50 \%$ maximum inhibitory concentration $\left(\mathrm{IC}_{50}\right)$ of filgotinib and spironolactone $(\sim 7 \mu \mathrm{M}$ and $4 \mu \mathrm{M}$, respectively) was within 3-fold of the plasma levels observed in clinical use (3.4 $\mu \mathrm{M}$ [ref. 65] and $1.5 \mu \mathrm{M}$ [ref. 66], respectively). Ruxolitinib and mycophenolic acid minimally suppressed HIV-1-dsGFP expression ( 0 and 0.2 log reduction, respectively) in these 3 cell lines.

We next examined the effect of these 4 HIV-1-suppressing agents on $\mathrm{CD} 4^{+} \mathrm{T}$ cells from virally suppressed HIV-1-infected individuals. We treated $\mathrm{CD} 4^{+} \mathrm{T}$ cells from virally suppressed HIV-1-infected individuals (Supplemental Table 2) with HIV-1suppressing agents for 24 hours and stimulated these cells with
PMA and ionomycin during the final 6 hours to test whether cells treated with HIV-1-suppressing agents can resist PMA/ionomycin-induced maximum latency reversal (ref. 67, Figure 1C, and Supplemental Figure 3). We determined HIV-1 expression levels using cell-associated HIV-1 RNA expression from aliquots of 1 million $\mathrm{CD}^{+} \mathrm{T}$ cells. We found that filgotinib, ruxolitinib, spironolactone, and mycophenolic acid significantly suppressed cell-associated HIV-1 RNA expression despite PMA/ionomycin challenge by $1.0 \mathrm{log}, 0.6 \mathrm{log}, 1.5 \mathrm{log}$, and $0.4 \mathrm{log}$, respectively $(P<0.05)$, ex vivo (Figure $1 C)$. Overall, we found that filgotinib and spironolactone inhibited HIV-1 expression more prominent- 
ly, both in cell line models and in $\mathrm{CD} 4^{+} \mathrm{T}$ cells from virally suppressed HIV-1-infected individuals.

Filgotinib preferentially suppresses spliced over unspliced HIV-1 transcription. To understand how HIV-1-suppressing agents reduce HIV-1 transcription, we examined whether it is the unspliced HIV-1 RNA, spliced HIV-1 RNA, or both that are affected. Using 3 HIV-1-infected cell line clones (Figure 3, A and B) and $\mathrm{CD} 4^{+} \mathrm{T}$ cells from virally suppressed HIV-1-infected individuals (Figure 3, C and D), we examined the expression levels of cell-associated total HIV-1 RNA (measuring polyadenylated HIV-1 RNA) (68), unspliced HIV-1 RNA (measuring gag RNA) (69), and spliced HIV-1 RNA (measuring tat/rev RNA) (70). We found that these HIV-1-suppressing agents changed spliced and unspliced HIV-1 RNA expression differently (Figure 3A). Among them, filgotinib suppressed spliced HIV-1 RNA expression more prominently than unspliced HIV-1 RNA expression (0.7 log vs. 0.2 log reduction in spliced vs. unspliced HIV-1 RNA, respectively) (Figure 3B). In contrast, the other JAK inhibitor, ruxolitinib, suppressed unspliced but not spliced HIV-1 RNA expression (0.1 log vs. 0.4 log reduction in spliced vs. unspliced HIV-1 RNA, respectively), while spironolactone (0.8 log vs. $0.8 \log$ reduction) and mycophenolic acid (0.4 log vs. $0.5 \log$ reduction) suppressed both spliced and unspliced HIV-1 RNA expression. We further examined the expression levels of total, unspliced, and spliced HIV-1 RNA in $\mathrm{CD}^{+} \mathrm{T}$ cells from virally suppressed HIV-1-infected individuals challenged with PMA and ionomycin (Figure 3C). We found that filgotinib suppressed the expression of spliced HIV-1 RNA preferentially compared with unspliced HIV-1 RNA (3.8 log vs. 0.2 log reduction in spliced vs. unspliced HIV-1 RNA, respectively) (Figure 3D). Notably, the low level of tat/rev expression in $\mathrm{CD}^{+}$cells from ART-suppressed HIV-1-infected individuals (18) may not provide sufficient dynamic range to determine whether HIV-1-suppressing agents reduce spliced HIV-1 RNA transcription, and quantification of tat/rev spliced HIV-1 RNA does not capture all spliced HIV-1 RNA species. Therefore, we compared the changes of total HIV-1 RNA and unspliced HIV-1 RNA, which can be readily captured and compared. We found that total HIV-1 RNA level significantly decreased in filgotinib-treated samples (average of $0.9 \log$ reduction, $P=$ 0.02), but unspliced HIV-1 RNA did not significantly decrease (average of $0.2 \log$ reduction, $P=0.4$ ), indicating that the decrease in total HIV-1 RNA was due to a decrease in spliced HIV-1 RNA, consistent with our findings in cell line models. This suggests that filgotinib affects HIV-1 RNA splicing through previously unknown mechanisms different from JAK inhibition (see below).

Filgotinib suppresses HIV-1-driven aberrant host gene transcription. The hallmark of HIV-1-driven aberrant host gene transcription at the integration site is HIV-1-to-host RNA splicing and high levels of host gene transcription downstream but not upstream of the HIV-1 integration site (71). In this event, the host gene transcription is controlled by HIV-1 promoter activity, not host immune homeostasis. When HIV-1 proviruses are integrated into proliferation-related genes, HIV-1-driven aberrant host gene transcription leads to excessive and unchecked proliferation of gene expression as a mechanism for HIV-1 integration site-dependent proliferation (71). We hypothesized that inhibition of HIV-1 transcription or splicing by HIV-1-suppressing agents can disrupt HIV-1-driven aberrant host gene transcription. To this end, we examined HIV-1-driven aberrant host gene transcription at the integration site upon treatment with HIV-1-suppressing agents. We used a cell line model (HIV-1-infected Jurkat clone 8B10) in which HIV-1-dsGFP reporter is integrated into the intron of a proliferation-related proto-oncogene, VAV1 (71). Integration into VAV1 is associated with clonal expansion of the HIV-1-infected cells in HIV-1-infected individuals (72) and in lentivirus-transduced CAR T cells (73), suggesting that integration into VAV1 is a clinically relevant mechanism driving integration site-dependent proliferation in vivo. In this cell line clone, HIV-1 drives high levels of aberrant VAV1 gene expression through HIV-1 promoter-driven expression and HIV-1-to-VAV1 splicing (71), particularly downstream but not upstream of the HIV-1 integration site (red arrowheads, Figure 4A). This reporter system provides a scalable measurement of HIV-1-driven aberrant host gene expression at the integration site. In mock-treated (DMSO) HIV-1-infected Jurkat clone 8B10, VAV1 RNA peaks downstream of the HIV-1 integration site are more than 6 times higher than the RNA peaks upstream of the HIV-1 integration site. Treatment with HIV-1-suppressing agents, particularly filgotinib, spironolactone, and mycophenolic acid, reduced HIV-1-driven aberrant VAV1 transcription (Figure 4A). To quantify the effect of HIV-1-suppressing agents on HIV-1driven aberrant host gene expression, we measured cellular canonical splicing (as measured by RNA reads capturing splicing between VAV1 exon 1 and exon 2), HIV-1-driven aberrant splicing (as measured by HIV-1-to-host chimeric RNA reads), and HIV-1 RNA expression (as measured by all HIV-1 RNA reads) in the HIV-1infected Jurkat clone 8B10 (Supplemental Figure 4). We found that filgotinib, but not other HIV-1-suppressing agents, significantly suppressed HIV-driven aberrant splicing to the host RNA by $1 \log$ (Supplemental Figure 4). Our results demonstrate that the reduction of HIV-1-driven aberrant host gene transcription by filgotinib is mediated through modulation of aberrant splicing.

At the protein expression level, HIV-1-driven aberrant VAV1 transcription led to a truncated VAV1 protein expression (Figure $4 \mathrm{~B}, \triangle \mathrm{VAV} 1$ ). This is because HIV-1 integrates downstream of the VAV1 translation start site and obliterates the N-terminal VAV1 protein coding regions. This $\mathrm{N}$-terminal VAV1 truncation removes the regulatory region and increases the oncogenicity of this oncogene (74). Western blot analysis showed that filgotinib, spironolactone, and mycophenolic acid treatment suppressed the oncogenic truncated VAV1 expression (Figure 4, B and C). Overall, we showed that HIV-1-suppressing agents not only suppress HIV-1 transcription itself but also disrupt HIV-1-driven aberrant host gene expression at the integration site. To our knowledge, this is the first study showing that HIV-1-driven aberrant host gene expression, a mechanism mediating HIV-1-integration site-dependent proliferation, can be disrupted by FDA-approved drugs.

Transcriptional landscape analysis identifies distinct effect of filgotinib on RNA processing. To understand the mechanisms of how HIV-1-suppressing agents suppress HIV-1 RNA expression, we performed transcriptome analysis using 3 independent methods: (a) Ingenuity Pathway Analysis (IPA) focusing on differential expression of genes reaching significant statistical difference (75), (b) gene set enrichment analysis (GSEA) focusing on whether an a priori-defined set of genes concordantly differ between 2 biological states using genome-wide profiling (76), and (c) exon-intron 


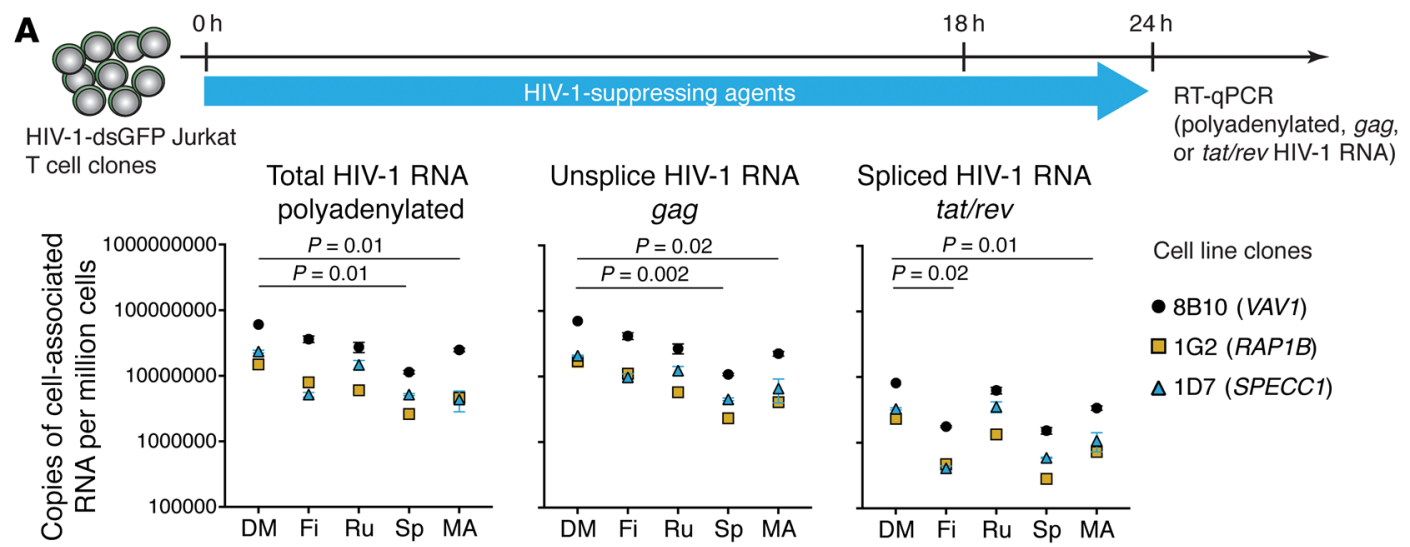

B
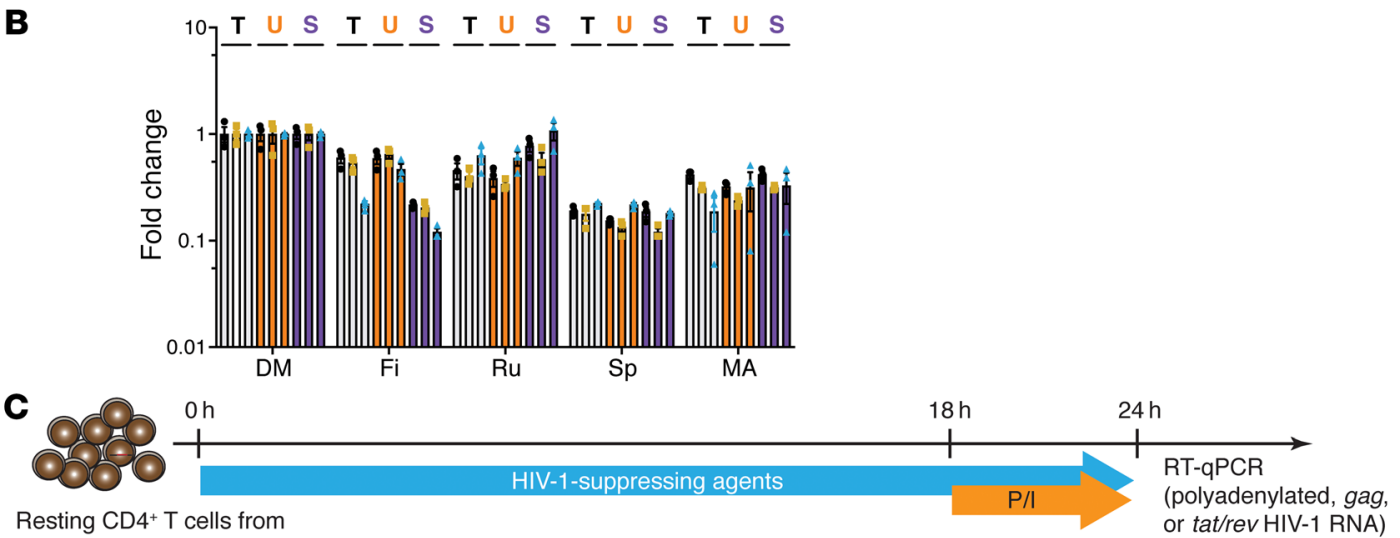

HIV-1-infected individuals
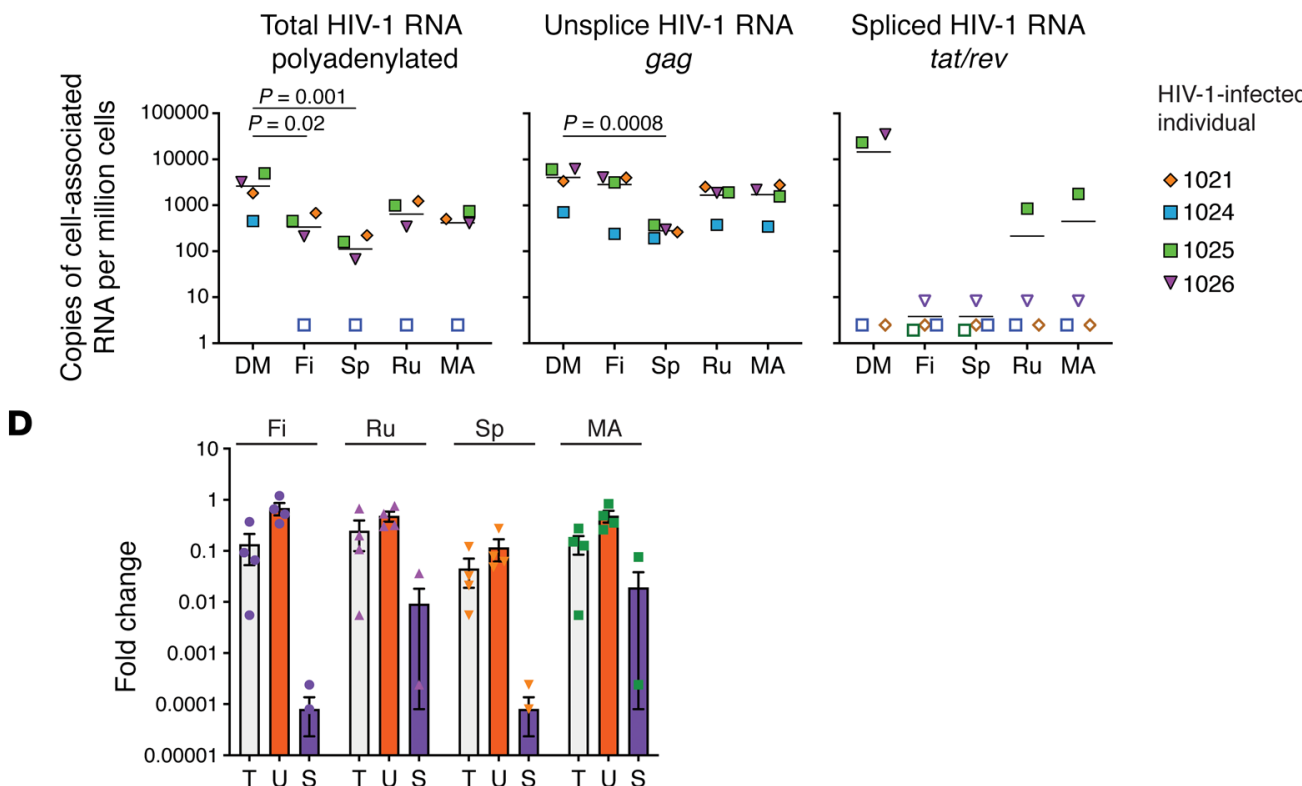

Figure 3. HIV-1-suppressing agents reduce different levels of spliced and unspliced HIV-1 RNA transcription. Cell-associated RNA levels (A and C) and fold inhibition (B and D) of total (polyadenylated) (68), unspliced ( $\mathrm{gag}$ (69), and spliced (tat/rev) (70) HIV-1 RNA in 3 HIV-1-dsGFP-Jurkat clones (A and B) and $\mathrm{CD}^{+} \mathrm{T}$ cells from virally suppressed HIV-1-infected individuals (C and $\left.\mathbf{D}\right)$ were measured by RT-qPCR. Cells were treated with HIV-1-suppressing agents (10 $\mu \mathrm{M}$ for 24 hours). In clinical samples (C and $\mathbf{D})$, aliquots of 1 million cells were treated with PMA/ionomycin challenge (for 6 hours) in the presence of ART ( $1 \mu \mathrm{M}$ tenofovir and $10 \mu \mathrm{M}$ enfuvirtide). $P$ values were calculated by Friedman's nonparametric ANOVA test (2-tailed) with uncorrected Dunn's test for comparison between each treatment and DMSO control. Error bars represent SEM from quadruplicates. DM, DMSO; Fi, JAK1 inhibitor filgotinib; Ru, JAK1/2 inhibitor ruxolitinib; Sp, DNA helicase inhibitor spironolactone; MA, IMPDH inhibitor mycophenolic acid; T, total HIV-1 RNA; U, unspliced HIV-1 RNA; S, spliced HIV-1 RNA. 
A

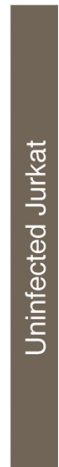

DMSO

Filgotinib

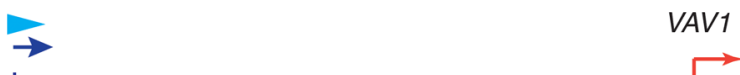

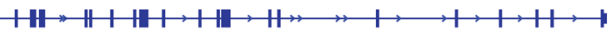

Ruxolitinib

1

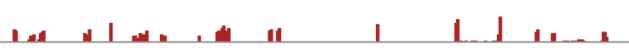

1

U1, III, I. II

1 1111

Spironolactone

Mycophenolic

acid

DMSO

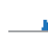

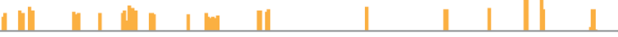

1

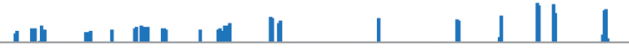
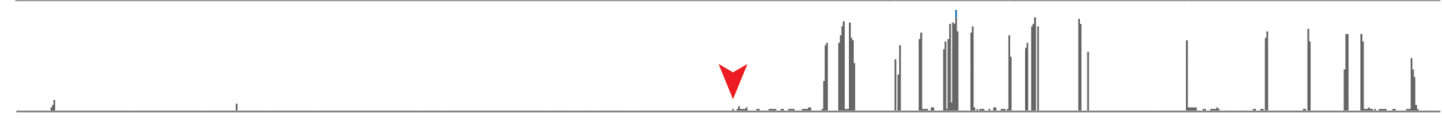

Filgotinib

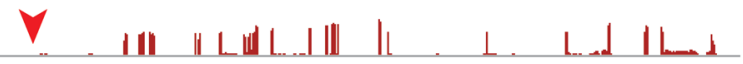

Ruxolitinib

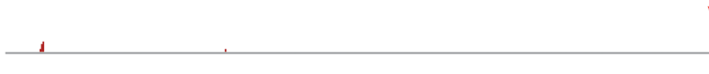

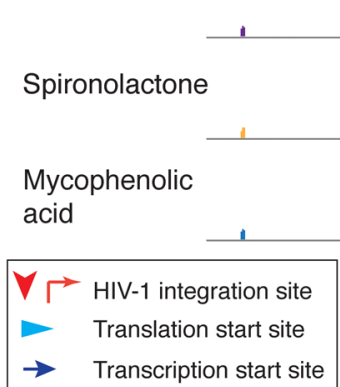

B

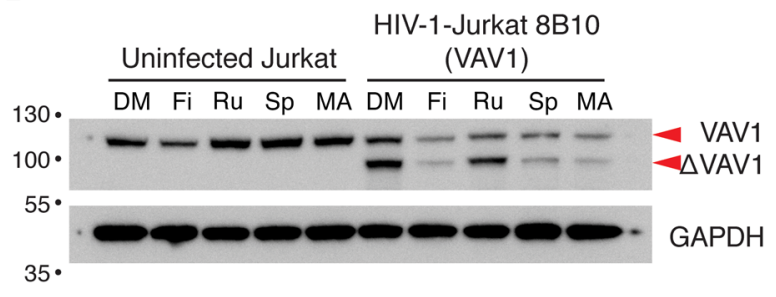

C

VAV1 in uninfected VAV1 in HIV-1. Jurkat Jurkat 8B10

$\triangle$ VAV1 in HIV-1Jurkat 8B10

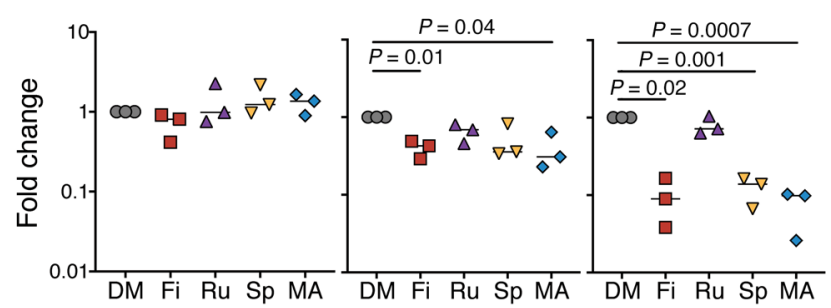

Figure 4. HIV-1-suppressing agents reduce HIV-1-driven aberrant host gene transcription at the integration site. (A) Normalized transcriptional landscape using first exon at the HIV-1 integration site (proto-oncogene VAV1) in the HIV-1-Jurkat clone 8 B10 treated with HIV-1-suppressing agents (10 $\mu$ M) for 24 hours. HIV-1 integration into VAV1 has been previously reported in CD4 ${ }^{+} T$ cells from virally suppressed HIV-1-infected individuals (72). Red arrowheads, HIV-1 integration site mapped by inverse PCR (58). (B) Western blot of VAV1 protein expression in HIV-1-infected Jurkat clone 8 B10 treated with HIV-1suppressing agents. (C) Relative quantification of intact VAV1 and truncated VAV1 expression to DMSO treatment normalized to GAPDH in triplicates. DM, DMSO; Fi, filgotinib; Ru, ruxolitinib; Sp, spironolactone; MA, mycophenolic acid. $P$ value was calculated with 1-way ANOVA with Fisher's least significant difference test for comparison between each treatment and DMSO control.

landscape mapping, which captures genes affected by changes in RNA splicing (77). Different from overexpression or downregulation of individual cellular factors, GSEA allows genome-wide understanding of how drug treatment affects the cellular environment. We compared the transcriptome of the HIV-1-infected 8B10 Jurkat cell clone (Supplemental Figure $5 \mathrm{~A}$ ) and CD4 ${ }^{+} \mathrm{T}$ cells from virally suppressed HIV-1-infected individuals (Figure 5A) treated with HIV-1-suppressing agents for 24 hours versus the transcriptome of mock-treated (DMSO) cells.
IPA provides standard examination of cellular genes up- or downregulated during pharmacological perturbations. Using IPA, we found that differentially expressed genes in filgotinib-treated cells, in particular, were strikingly enriched in RNA splicing and RNA processing (Supplemental Figure 5B and Figure 5B), which was not seen in cells treated with other HIV-1-suppressing agents (Supplemental Figure 6).

GSEA, unlike IPA, does not involve filtering out up- or downregulated individual genes based on an arbitrary false discovery 
A Differentially expressed (DE) genes of $\mathrm{CD4}^{+}$cells from HIV-1-infected individuals

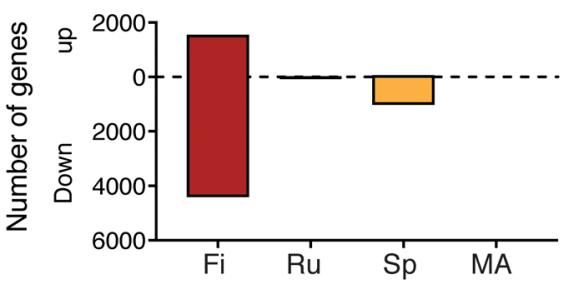

B Disease and biological function of DE genes of $\mathrm{CD}^{+}{ }^{+}$cells from HIV-1-infected individuals

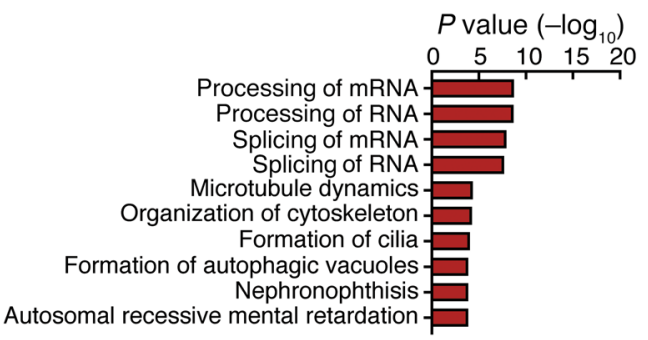

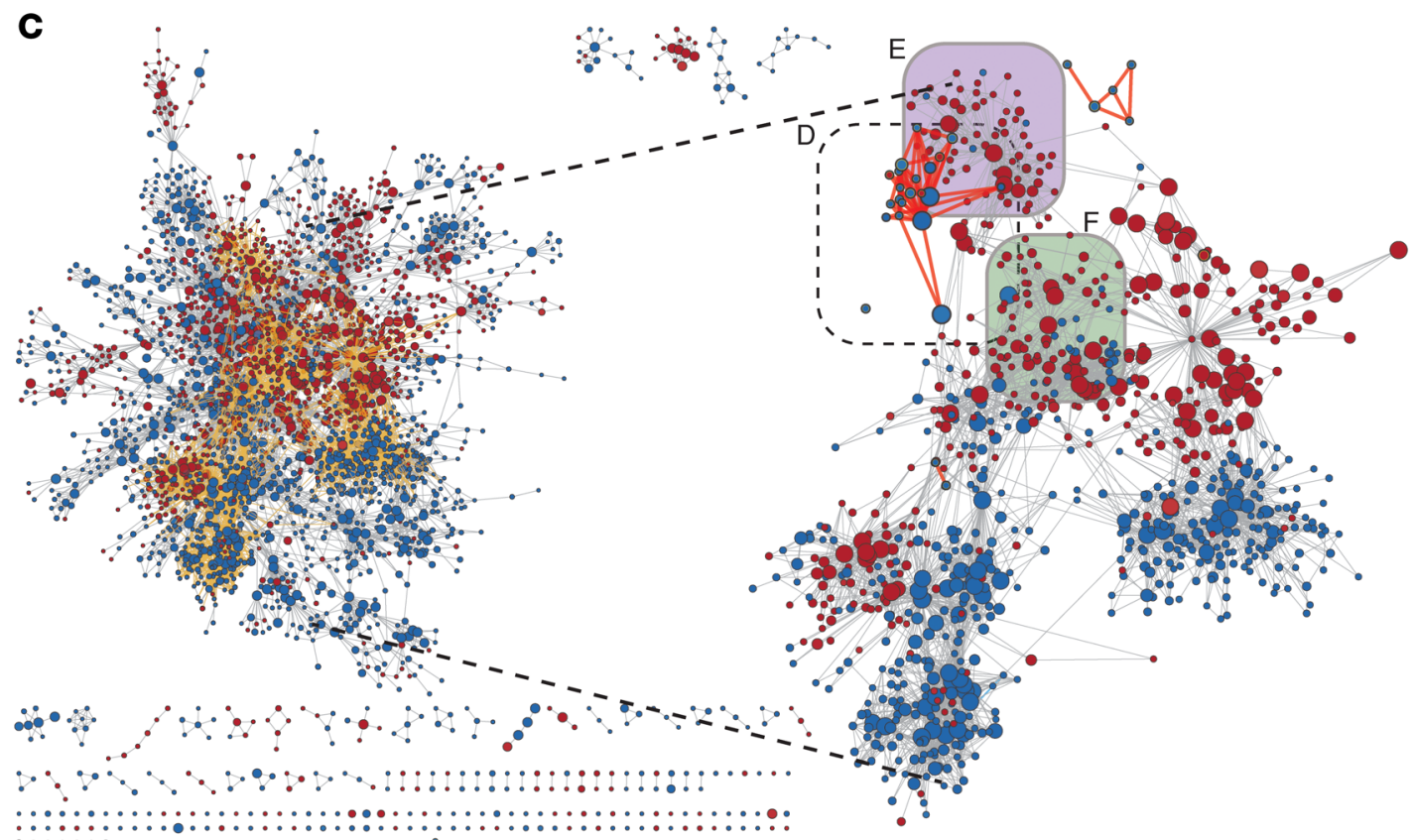
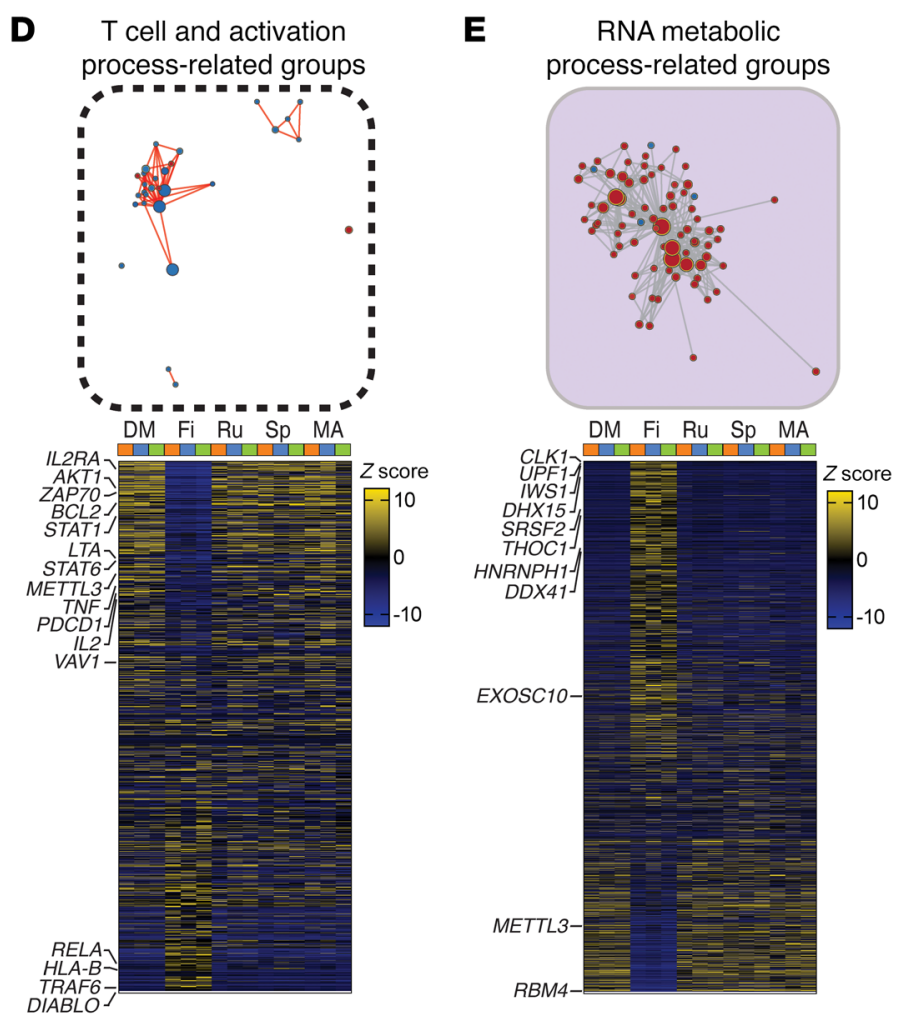

F Chromatin organizationrelated groups
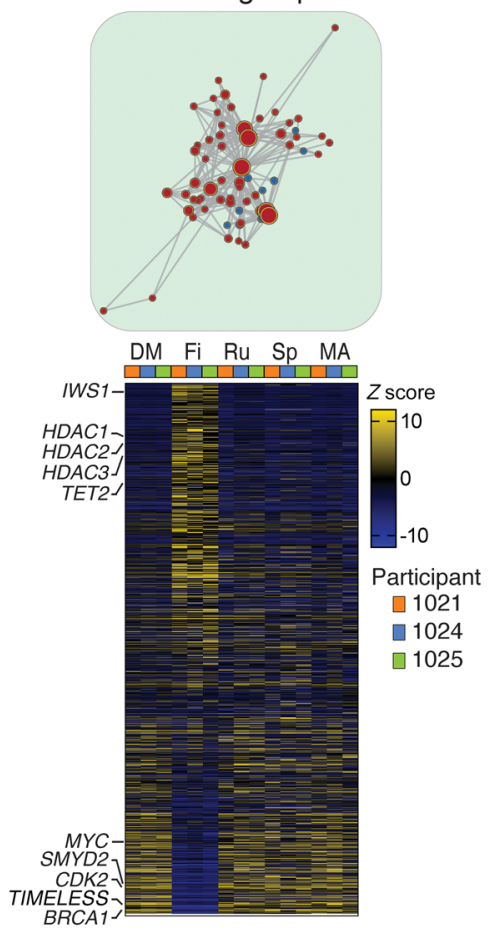
Figure 5. Pathway enrichment analysis of $\mathrm{CD4}^{+} \mathrm{T}$ cells from virally suppressed HIV-1-infected individuals treated with HIV-1-suppressing agents ex vivo. (A) Differential gene expression analysis of $\mathrm{CD} 4^{+} \mathrm{T}$ cells from 3 virally suppressed HIV-1-infected individuals (participants 1021, 1024 , and 1025) treated with $10 \mu \mathrm{M}$ HIV-1-suppressing agents for 24 hours in the presence of ART ( $1 \mu \mathrm{M}$ tenofovir and $10 \mu \mathrm{M}$ enfuvirtide). (B) Disease and biological function pathway analysis of differentially expressed genes using Ingenuity Pathway Analysis (IPA). (C) Results of GSEA (76) with Gene Ontology (GO) gene sets visualized by EnrichmentMap in Cytoscape $(79,114)$. Each node represents one GO gene set in the GSEA Molecular Signature Database (MSigDB) that is significantly enriched (FDR $<0.25$ ). The size of the node represents the number of genes in each gene set. Red represents a positive enrichment score, and blue represents a negative enrichment score. The edge (connected lines between nodes) represents the degree of gene overlap between nodes. The cutoff of overlap coefficient is 1 . We identified the most enriched pathways from the top 17 nodes with more than 50 interactions each. (D-F) The expression levels of T cell activation-related gene sets (D), RNA metabolic process-related genes (E), and chromatin organization-related genes $(\mathbf{F})$ were analyzed using $C D 4^{+} \mathrm{T}$ cells from 3 virally suppressed HIV-1-infected individuals. DM, DMSO; Fi, filgotinib; Ru, ruxolitinib; Sp, spironolactone; MA, mycophenolic acid.

rate (FDR) or $P$ value. Instead, GSEA examines the transcriptional levels of all the genes involved in candidate cellular pathways (gene sets) and determines whether certain cellular pathways are more prominently affected upon pharmacological perturbations. Using GSEA, we examined all Gene Ontology (GO) gene sets on the Molecular Signatures Database (MSigDB) (78) and visualized the pathway interaction networks using EnrichmentMap in Cytoscape (79). Using the highest overlap coefficient, we identified the most enriched pathways that may have pivotal influence in the cellular environment (Figure 5C and Supplemental Figure 5C). Among them, closely clustered GO gene sets represent overlapping genes, which highlight pathways occurring simultaneously.

We found that the transcriptome signature in filgotinib-treated cells is strikingly different from that in cells treated with the other JAK inhibitor, ruxolitinib, and other HIV-1-suppressing agents. First, as a JAK inhibitor, filgotinib suppressed T cell activation, demonstrating downregulation of the JAK/STAT signaling pathway (STAT6), PI3K/Akt pathways (AKT1), TCR signaling (ZAP7O and IL2RA [CD25]), and the exhaustion marker PDCD1 (PD1) (Supplemental Figure 5D and Figure 5D). While both filgotinib and ruxolitinib were JAK inhibitors, filgotinib-mediated reduction in $\mathrm{T}$ cell activation was significantly more prominent.

Second, consistent with IPA analysis (Figure 5B), we found that filgotinib-treated cells upregulated genes related to RNA processing, including RNA splicing (Figure 5E and Supplemental Figure $5 \mathrm{E})$. Importantly, many of these RNA processing genes have been reported to be critical for HIV-1 transcription, such as CLK1 $(80,81)$, UPF1 (82, 83), THOC1 (84), IWS1 (85), and METTL3 (86). Disrupted RNA expression of these HIV-1-related RNA processing genes may mediate preferential inhibition of HIV-1 splicing (Figure 3).

Third, we found that filgotinib-treated cells had altered chromatin organization and modification pathways (Supplemental Figure $5 \mathrm{~F}$ and Figure $5 \mathrm{~F}$ ) that have been reported to affect HIV-1 transcription, such as TET2 (87), HDAC1-HDAC3 (88-90), CDK2 (91, 92), SMYD2 (93), and BRCA1 (94). Notably, the class I histone deacetylases HDAC1-HDAC3, but not other HDACs, induce HIV-1 repression (95). Overall, filgotinib functioned not only as a JAK inhibitor that suppressed $\mathrm{T}$ cell activation but also inhibited cellular factors involved in HIV-1 transcription, splicing, and reactivation. Our findings demonstrate that genome-wide transcriptome analysis can systematically identify potential mechanisms mediating HIV-1 transcription and reactivation.

Filgotinib may potentially function as a splicing inhibitor that induces intron retention. Our finding that filgotinib suppressed spliced HIV-1 RNA expression more prominently than unspliced HIV-1 RNA expression (Figure 3) suggests that filgotinib functions as a splicing inhibitor. During normal cellular splicing events, introns in pre-mRNAs are spliced out, leaving mature mRNA that does not contain introns. When RNA splicing is disrupted, one of the key features is that introns remain in the mature mRNA, a phenomenon called intron retention. Intron retention has emerged as a mechanism of gene expression control $(96,97)$, in cellular function (98), cancer (99), and HIV-1 infection (100). We examined the impact of filgotinib on RNA splicing using intron retention as a surrogate from the same RNA-Seq data set. Using IRFinder (77), which systematically identifies and quantifies retained introns using bioinformatic calculations, we identified genes harboring retained introns and visualized the exon-intron landscape. Among the 4 HIV-1-suppressing agents, we found that only filgotinib induced a higher frequency of intron retention in both HIV-1-infected Jurkat clone $8 \mathrm{~B} 10$ and $\mathrm{CD} 4^{+} \mathrm{T}$ cells from virally suppressed HIV-1-infected individuals (Supplemental Figure 7A and Figure 6A). We found that genes harboring retained introns upon filgotinib treatment were enriched in RNA processing and splicing pathways (Supplemental Figure 7, B-H, and Figure 6B). In particular, filgotinib induced intron retention in key RNA processing-related genes in HIV-1 transcription, such as HNRNPH1 (101) and DDX 41 (ref. 102 and Figure 6, C-F, blue arrowheads). Intron retention in HNRNPH1 and DDX41 led to reduced protein expression (Figure 6G). Our results suggest that filgotinib may potentially function as a splicing inhibitor that induces intron retention in genes involving HIV-1 RNA processing.

Filgotinib reduces $T$ cell activation upon stimulation. Filgotinib is an immunomodulatory agent clinically used to suppress aberrant $\mathrm{T}$ cell activation in autoimmune diseases. We asked whether JAK inhibition suppresses T cell activation in HIV-1-infected individuals. We activated $\mathrm{CD} 4^{+} \mathrm{T}$ cells from virally suppressed HIV-1-infected individuals with CD3/CD28 stimulation for 4 days to induce $\mathrm{T}$ cell activation and cellular proliferation. The culture was supplemented with ART (tenofovir and enfuvirtide) to prevent new rounds of infection. Cells were treated with DMSO, filgotinib, ruxolitinib, spironolactone, or mycophenolic acid for 4 days. We found that the JAK inhibitors filgotinib and ruxolitinib and the immune modulator mycophenolic acid suppressed $\mathrm{T}$ cell activation (as measured by CD25 expression) and PD1 expression (Supplemental Figure 8), while spironolactone did not. Overall, we found that filgotinib suppressed $\mathrm{T}$ cell activation both at the transcription level (Figure 5D) and as measured by surface protein expression of activation markers (Supplemental Figure 8).

Filgotinib reduces the proliferation of HIV-1-infected cells upon $T$ cell activation. To test whether HIV-1-suppressing agents inhibit $\mathrm{T}$ cell proliferation, we tracked cellular proliferation upon CD3/ CD28 stimulation using CellTrace staining (Supplemental Figure 
A Intron-retained (IR) genes of CD4+ cells from HIV-1-infected individuals

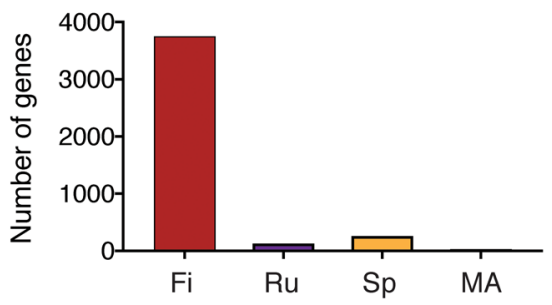

HIV-1-infected Jurkat 8B10

C

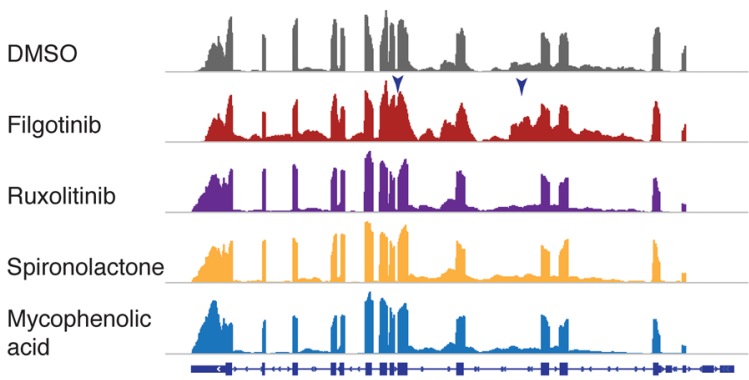

E

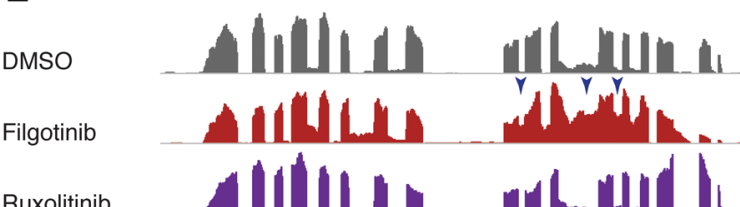

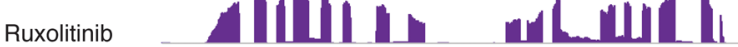
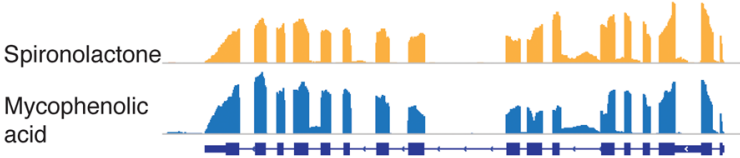

$\checkmark$ Intron retention

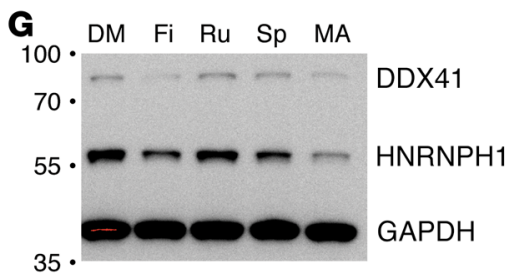

B Disease and biological function of IR genes of $\mathrm{CD}^{+}{ }^{+}$cells from HIV-1-infected individuals

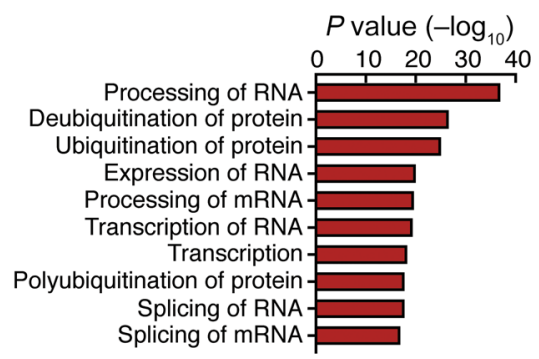

HIV-1-infected individuals

D HNRNPH1

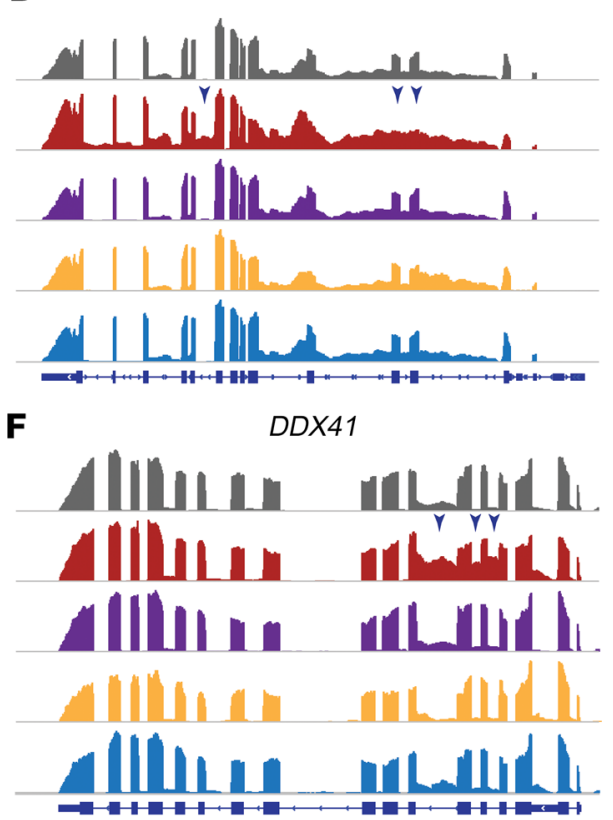

Figure 6. Filgotinib induces intron retention in RNA processing-related genes in both HIV-1-infected Jurkat clone 8B10 and CD4+ T cells from virally suppressed HIV-1-infected individuals. (A) The number of intron-retained genes identified by IRFinder (77) in CD4 ${ }^{+}$T cells treated with HIV-1-suppressing agents using samples described in Figure 5. CD4+ $T$ cells were obtained from 3 virally suppressed HIV-1-infected individuals (participants 1021, 1024, and 1025) treated with $10 \mu \mathrm{M} \mathrm{HIV-1-suppressing} \mathrm{agents} \mathrm{for} 24$ hours in the presence of ART ( $1 \mu \mathrm{M}$ tenofovir and $10 \mu \mathrm{M}$ enfuvirtide). Intron-retained regions with significantly increased ratio $(P<0.05$, unequal-variances $t$ test) are reported. (B) Disease and biological function pathway analysis of intron-retained genes using IPA in $\mathrm{CD}^{+} \mathrm{T}$ cells from 3 virally suppressed HIV-1-infected individuals treated with filgotinib. (C-F) Integrative genome viewer plots of representative RNA landscape of HNRNPH1 and DDX41 demonstrate intron retention in these genes (blue arrowheads) in HIV-1-dsGFP Jurkat clone 8B10 (C and E) and in $C D 4^{+}$T cells from virally suppressed HIV-1-infected individuals (D and F). (C) Western blot of intron-retained genes HNRNPH1 and DDX41 in HIV-1infected Jurkat 8B10 cells treated with HIV-1-suppressing agents. DM, DMSO; Fi, filgotinib; Ru, ruxolitinib; Sp, spironolactone; MA, mycophenolic acid.

8). While mycophenolic acid halted proliferation of all CD $4^{+} \mathrm{T}$ cells ( $<1 \%$ proliferated), the JAK inhibitor filgotinib had a modest inhibitory effect on proliferation of $\mathrm{CD} 4^{+} \mathrm{T}$ cells ( $\sim 4 \%$ proliferation).

To test whether HIV-1-suppressing agents preferentially suppress proliferation of HIV-1-infected cells over uninfected cells, we measured the frequency of cells harboring inducible HIV-1 by the end of CD3/CD28 stimulation. After 4 days of CD3/CD28 stimulation, we cultured the cells in limiting dilution for 2 days to allow the effect of HIV-1-suppressing agents to be washed out. To determine whether a 2-day washout period is sufficient to remove HIV-1- 

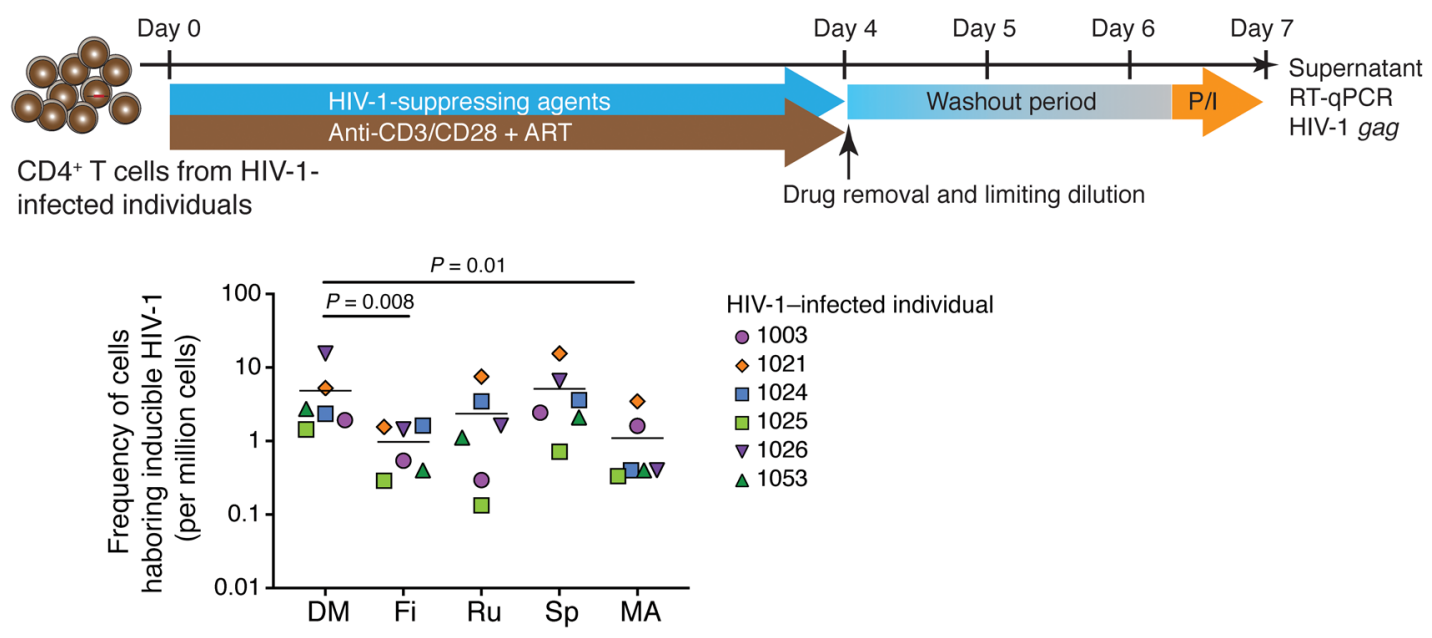

Figure 7. HIV-1-suppressing agents reduce the frequency of cells harboring inducible HIV-1. CD4+ $\mathrm{T}$ cells from 6 virally suppressed HIV-1-infected individuals were activated with anti-CD3/CD28 antibodies for 4 days in the presence of HIV-1-suppressing agents (10 $\mu \mathrm{M})$ and ART ( $1 \mu \mathrm{M}$ tenofovir and $10 \mu \mathrm{M}$ enfuvirtide) to allow cellular proliferation without new rounds of infection. Cells were plated at limiting dilution (200,000 cells per well) to calculate the frequency of cells harboring inducible HIV-1. After 2 days allowing washout of the HIV-1-suppressing agents, cells were stimulated with PMA/ionomycin to induce HIV-1 RNA expression. The use of inducible HIV-1 RNA assay by measurement of supernatant HIV-1 RNA allows a wide dynamic range to calculate the frequency of cells harboring inducible HIV-1. $P$ values were calculated by Friedman's nonparametric ANOVA test (2-tailed) with uncorrected Dunn's test for comparison between each treatment and DMSO control. DM, DMSO; Fi, filgotinib; Ru, ruxolitinib; Sp, spironolactone; MA, mycophenolic acid.

suppressing effects, we examined HIV-1-driven GFP expression in the HIV-1-infected Jurkat clone 8 B10 after removal of HIV-1suppressing agents. We found that HIV-1-driven GFP expression returned to basal level within 2 days after drug removal (Supplemental Figure 9). By measuring the frequency of HIV-1-infected cells over total cells using limiting-dilution culture after drug treatment, we would be able to examine whether HIV-1-suppressing agents preferentially reduce proliferation of HIV-1-infected cells over total cells. We then stimulated the cells with PMA and ionomycin to induce maximal latency reversal and measured the frequency of cells harboring inducible HIV-1 using supernatant HIV-1 RNA detection $(103,104)$. While supernatant HIV-1 RNA expression does not equal the presence of replication-competent HIV-1 (28), this cell-free RNA-based inducible RNA assay provides a wide dynamic range to measure the frequency of cells harboring inducible HIV-1 with readouts correlating with quantitative viral outgrowth cultures (ref. 103 and Figure 7). We found that filgotinib and mycophenolic acid significantly reduced the frequency of cells harboring inducible HIV-1 (0.6 log and $0.7 \log$ reduction, respectively). Overall, we found that filgotinib reduced $\mathrm{T}$ cell activation and the proliferation of HIV-1-infected cells.

\section{Discussion}

The combination of drug screen and 3 levels of transcriptome analysis (based on differential expression, gene set enrichment, and exon-intron landscape analysis) systematically identified what we believe are novel cellular pathways required for HIV-1 transcription as druggable therapeutic targets. Our drug screen not only confirmed previously reported HIV-1 transcription inhibitors but also identified an HIV-1-suppressing agent, filgotinib. Our transcriptome analysis broadened our understanding of drug effects by genome-wide identification of all pathways involved (Figure 5). Using these transcriptome analyses, we found that fil- gotinib is not merely a JAK inhibitor. We found a function of filgotinib as a splicing inhibitor, modulating HIV-1 RNA splicing and transcription-related cellular factors and mediating preferential suppression of HIV-1 RNA splicing.

We systematically examined the landscape of cellular pathways involved in HIV-1 transcription and respective FDAapproved drugs that target these pathways (Figure 2). We found that both $\mathrm{T}$ cell activation pathways and HIV-1 transcription are necessary for full HIV-1 reactivation. We identified additional T cell activation-related pathways that can be inhibited to suppress HIV-1 transcription through the use of JAK/STAT inhibitors (filgotinib and ruxolitinib), tyrosine kinase inhibitors (dovitinib, pazopanib, and ponatinib), Akt inhibitors (uprosertib), and cation transporter inhibitors (digoxin [ref. 105], levosimendan [ref. 45], and zinc pyrithione). More importantly, we identified RNA transcription pathways involved in HIV-1 transcription, including transcription initiation, elongation, splicing, and nuclear export. Transcription initiation requires DNA unwinding by the DNA helicase TFIIH (106) and topoisomerases (107) to relieve the supercoiling tension during transcription, which can be inhibited by the DNA unwinding inhibitors irinotecan, mitoxantrone, and mitomycin $\mathrm{C}$ or by the DNA helicase inhibitor spironolactone (50). RNA synthesis requires IMPDH for de novo GTP synthesis, which can be inhibited by mycophenolic acid (51). Transcription elongation requires cyclin-dependent kinases (CDKs), which can be inhibited by the CDK inhibitor flavopiridol. RNA splicing can be inhibited by filgotinib. Finally, nuclear export of unspliced HIV-1 RNA can be inhibited by the CRM1 inhibitor KPT-330 (32). We found that filgotinib, as a JAK inhibitor, suppresses genes related to T cell activation such as IL2RA, AKT1, STAT1, VAV1, and $P D C D 1$ (Figure 5) as expected. We found that filgotinib affects HIV-1 and cellular RNA processing, which can be considered as a new function or an off-target effect. We postulate that this may 
be because filgotinib, which binds to the ATP binding site in JAK, also binds to RNA processing enzymes. This suggests that combinatorial strategies targeting HIV-1 reactivation pathways can be achieved by one single drug. HIV-1-suppressing agents identified in this study, as a proof of concept, can serve as lead compounds for further development of HIV-1-specific therapeutic strategies, likely in combinatorial approaches.

Despite the essential role of RNA splicing $(32,108,109)$ in HIV-1 life cycle and pathogenesis, little is known about how targeting HIV-1 RNA splicing can be applied as a therapeutic strategy in the context of HIV-1 persistence (110). Studies have shown that HIV-1 splicing and HIV-1-induced aberrant splicing are associated with HIV-1 persistence $(71,111,112)$, which makes RNA splicing an important potential therapeutic target. In contrast to traditional differential gene expression analysis, which identifies a list of upand downregulated genes, we broadened transcriptome analysis into qualitative visualization of the RNA transcription landscape. Using RNA landscape approaches, we found that HIV-1-driven cancer-related gene expression can be suppressed by HIV-1-suppressing agents (Figure 4), and we found potentially novel functions of filgotinib as a splicing inhibitor (Figure 6). These findings in HIV-1-host interactions and HIV-1-drug interactions cannot be found by traditional transcriptome analyses. Our findings highlight the importance of HIV-1 and cellular RNA landscape analysis in studying HIV-1-host interactions and identifying previously unknown mechanisms of drug effects.

Overall, a combination of drug screening and transcriptome analysis identified the landscape of cellular pathways critical for HIV-1 reactivation, and an HIV-1-suppressing agent, filgotinib. Filgotinib suppresses HIV-1 transcription and reduces the proliferation of HIV-1-infected cells by targeting 2 different pathways, involving inhibition of $\mathrm{T}$ cell activation and modulation of HIV-1 splicing. Therapeutic strategies targeting a combination of these pathways with increased selectivity against HIV-1-infected cells provide a new direction to reduce HIV-1-related immune activation and the proliferation of HIV-1-infected cells.

\section{Methods}

\section{Clinical sample processing}

Resting $\mathrm{CD}^{+}{ }^{+} \mathrm{T}$ cells from ART-treated, virally suppressed HIV-1infected individuals were isolated using magnetic negative depletion (EasySep human CD4 ${ }^{+} \mathrm{T}$ cell enrichment kit [STEMCELL, 17952], CD69 MicroBead Kit, CD25 MicroBead Kit, and HLA-DR MicroBead Kit [Miltenyi Biotec]).

\section{Construction of Jurkat T cell clones containing known HIV-1 reporter provirus integration site}

Jurkat T cells (NIH AIDS Reagent Program) were transduced with a single-round HIV-1 reporter virus, NL4-3-d6-dsEGFP (42), at a low multiplicity of infection ( $<1 \%$ GFP expression). Briefly, Env in the fulllength NL4-3 genome was replaced by a destabilized EGFP. Inactivating point mutations were introduced into viral genes except for tat and rev. This reporter retains TAR, RRE, and all splice elements of the NL4-3 backbone. GFP-positive cells were sorted 3 days after infection into single cells and grown into clones. Cell line clones that grew into visible pellets were collected for expansion after 3 weeks. Integration sites of these HIV-1-dsGFP-Jurkat T cell clones were examined using inverse PCR $(58,113)$. These clones express various levels of HIV-1driven GFP due to stochastic activation via a Tat feedback loop (16) and can be induced to nearly $100 \%$ of GFP expression upon PMA $(50 \mathrm{ng} / \mathrm{mL}) /$ ionomycin $(1 \mu \mathrm{M})$ stimulation. For dual-reporter Jurkat clones, HIV-1-dsGFP-Jurkat clones were transduced with EF1 $\alpha$-driven destabilized BFP containing a nuclear localization signal and sorted by BFP positivity 3 days after transduction.

\section{HIV-1-suppressing agents screen}

A small-molecule compound library of 1,430 FDA-approved smallmolecule compounds (Selleckchem, L1300) were aliquoted to seventeen 96-well plates for a final concentration of $10 \mu \mathrm{M}$ for each compound. In each 96-well plate, 4 wells of flavopiridol served as positive controls and 4 wells of DMSO served as negative controls. Cells were fixed and examined under flow cytometry for GFP expression 24 hours after drug treatment.

\section{Drug treatment condition and assays}

Dose-response curves of HIV-1-dsGFP-Jurkat clones in vitro. All drug treatment was performed at $10 \mu \mathrm{M}$ unless otherwise specified. Quadruplicates of 100,000 uninfected Jurkat cells or HIV-1-infected Jurkat T cell clones were seeded in 96-well plates with DMSO or $0.01 \mu \mathrm{M}$, $0.1 \mu \mathrm{M}, 1 \mu \mathrm{M}, 2.5 \mu \mathrm{M}, 5 \mu \mathrm{M}$, and $10 \mu \mathrm{M}$ HIV-1-suppressing agents for 24 hours. Cells were stained with LIVE/DEAD Fixable Near-IR Dead Cell Stain Kit (Thermo Fisher Scientific, L10119) and fixed for flow cytometric analysis.

Immune phenotype of $\mathrm{CD}^{+} \mathrm{T}$ cells treated with HIV-1-suppressing agents. $\mathrm{CD} 4^{+} \mathrm{T}$ cells from virally suppressed HIV-1-infected individuals were stained with CellTrace Violet (Thermo Fisher Scientific, C34571) and activated with Dynabeads human T-activator CD3/CD28 (Thermo Fisher Scientific), IL-2 (30 U/mL), tenofovir $(1 \mu \mathrm{M})$, and enfuvirtide $(10 \mu \mathrm{M})$, and respective HIV-1-suppressing agents (filgotinib, ruxolitinib, spironolactone, and mycophenolic acid) $(10 \mu \mathrm{M})$ or DMSO for 4 days. Cells were stained with anti-CD25-BV605 (clone 2A3, BioRad, 562660), anti-PD1-APC (clone J105, Thermo Fisher Scientific, 17-2799-42), and LIVE/DEAD Fixable Near-IR Dead Cell Stain Kit (Thermo Fisher Scientific, L10119) for flow cytometric analysis.

RT-qPCR of resting $\mathrm{CD}^{+} \mathrm{T}$ cells from HIV-1-infected individuals treated with drugs ex vivo and HIV-1-dsGFP-Jurkat clones. Cells treated with HIV-1-suppressing agents for 24 hours were lysed in TRIzol ( 1 million cells in $300 \mu \mathrm{L}$ ), and total RNA was extracted with Direct-zol RNA Miniprep kits (Zymo Research, R2051). Total (68), unspliced (69), and spliced (70) HIV-1 RNA or cellular RNA (POLR2A) was measured by quantitative reverse transcription PCR (RT-qPCR) using qScript XLT 1-Step RT-qPCR ToughMix LowROX (Quanta Bio, 95134-02K) or TaqMan Gene Expression assays (Thermo Fisher Scientific, Hs00172187_m1), respectively. Primers and probes for total, unspliced, and spliced HIV-1 RNA were as follows: total RNA (primers, 5'-CAGATGCTGCATATAAGCAGCTG-3' and $5^{\prime}$-TTTTTTTTTTTTTTTTTTTTTGAAGCACTC-3'; probe, 5'FAM-CCTGTACTGGGTCTCTCTGG-Q 3') (68); gag (primers, $5^{\prime}$-CATGTTTTCAGCATTATCAGAAGGA-3' and 5'-TGCTTGATGTCCCCCCACT-3'; probe, 5'FAM-CCACCCCACAAGATTTAAACACCATGCTAA-Q $3^{\prime}$ ); tat/rev (primers, 5'-CTTAGGCATCTCCTATGGCAGGA-3' and 5'-GGATCTGTCTCTGTCTCTCTCTCCACC-3'; probe, 5'FAM-AGGGGACCCGACAGGCCC-Q3'). 
Quantification of induced HIV-1 RNA. CD4 ${ }^{+} \mathrm{T}$ cells from virally suppressed HIV-1-infected individuals were treated with Dynabeads human T-activator CD3/CD28 (Thermo Fisher Scientific, 111.32D), IL-2 $(30 \mathrm{U} / \mathrm{mL})$, tenofovir $(1 \mu \mathrm{M})$, and enfuvirtide $(10 \mu \mathrm{M})$, and respective HIV-1-suppressing agents (filgotinib, ruxolitinib, spironolactone, and mycophenolic acid) $(10 \mu \mathrm{M})$ or DMSO for 4 days. Cells were then pelleted to remove HIV-1-suppressing agents and plated at limiting dilution (200,000 cells per well). After a 2-day washout period allowing the effect of HIV-1-suppressing agents to decline, cells were stimulated with PMA/ionomycin for 18 hours. Culture supernatant of each of the limiting-dilution wells was collected for RNA extraction and supernatant RT-qPCR of HIV-1 gag, as modified from an inducible HIV-1 RNA assay (103).

\section{RNA-Seq analysis}

RNA extraction and library construction. Samples treated with HIV-1suppressing agents for 24 hours were collected for total RNA isolation using TRIzol (Thermo Fisher Scientific) followed by Direct-zol RNA extraction kit (Zymo Research). Approximately 500 ng of total RNA was used to generate a cDNA library using a TruSeq mRNA Library Prep kit (Illumina) for Illumina HiSeq $40002 \times 100$ bp sequencing or an NEBNext Ultra II directional mRNA library prep kit (New England Biolabs) for Novaseq $60002 \times 150 \mathrm{bp}$ sequencing.

Trimming and alignment. Low-quality reads from raw FASTQ files were trimmed using Trimmomatic with "LEADING:3 TRAILING:3 SLIDINGWINDOW:4:30 MINLEN:50” arguments. Trimmed FASTQ files were aligned using STAR 2.6.1d or 2.7.0f (https://github.com/ alexdobin/STAR) with the following arguments: "--readFilesCommand gunzip -c --outSAMtype BAM SortedByCoordinate --chimOutType SeparateSAMold --runThreadN 20 --outFilterType BySJout --alignSJoverhangMin 5 --alignSJDBoverhangMin 3 --chimSegmentMin 15 --outSAMstrandField intronMotif --outSAMattributes All --quantMode GeneCounts", to customized human assembly hg38 release 91 plus HIV-1 sequence (HXB2 or HIV-1-dsGFP).

Pathway enrichment on differentially expressed genes. Total gene counts and normalization of STAR-aligned BAM files were obtained using HTseq (htseq-count -r pos -f bam -s reverse) and DESeq2 with human genome assembly hg38 release 91 . Genes with a sum of read counts smaller than 10 were filtered out. Differentially expressed genes were analyzed with an adjusted $P$ value less than 0.05 and fold change over $\log _{2}$ of 1.6 (3-fold). Disease and biological function pathway analysis was carried out using IPA with the following analysis setting: Species, Human; Tissues and Cell, Immune cells, other cells, other tissues and primary cells, immune cell lines, other cell line. The top 10 enriched pathways are presented in this article.

GSEA and visualization. Total gene counts and normalization of STAR-aligned BAM files were obtained using HTseq (htseq-count - $r$ pos - $\mathrm{f}$ bam -s reverse) and DESeq2 with human genome assembly hg38 release 91 . Genes with a sum of read counts smaller than 10 were filtered out. Normalized counts were analyzed using GSEA (4.0.2) desktop software (https://www.gsea-msigdb.org/gsea/index.jsp) with c5.all.v7.symbols.gmt (GO) gene sets database, number of permutations 1000, No_Collapse, phenotype of permutation type, and Human_ENSEMBL_Gene_ID_MSigDBv7.0.chip. The result was then visualized using EnrichmentMap in Cytoscape with FDR less than 0.25 , $P$ value less than 0.005 , overlap efficiency 1 . Top clustered gene sets were selected by selection of nodes that had the largest number of immediate neighbors.
Intron retention (IR) analysis. Quality trimmed FASTQ files were analyzed with IRFinder 1.2.5 using STAR-built indexes with human genome assembly hg38 release 91.gtf as reference annotation (77). Up to triplicates of treatments were compared. Output files were filtered with the following criteria in Rstudio (1.1.463): 'A-IRratio' >= 0.1 \& 'A-IntronCover' $>=0.7 \&$ 'A-IntronDepth' $>=6$ \& 'A-SplicesExact' $>=2$ \& 'B-IRratio' $<0.1$ \& 'B-SplicesExact' $>=2$. Unequal-variances $t$ test of IRratio between 2 treatment groups was applied, and intronretained regions with significant difference $(P<0.05)$ were reported.

\section{Western blot}

Anti-VAV1 antibody (clone 2E11, Thermo Fisher Scientific, MA517198), anti-GAPDH antibody (clone GA1R, Thermo Fisher Scientific, MA5-15738), anti-HNRNPH1 antibody (clone OTI4F10, Thermo Fisher Scientific, MA5-27375), and anti-DDX41 antibody (clone C-3, Santa Cruz Biotechnology, sc-166225) were used in this study.

\section{Data and materials availability}

RNA-Seq results are available at the NCBI's Gene Expression Omnibus database (GEO GSE149353).

\section{Statistics}

We first performed normality testing using Shapiro-Wilk and Kolmogorov-Smirnov tests in GraphPad Prism. Samples treated in independent culture wells were each compared with controls and were not corrected by multiple comparisons. For HIV-1 RNA copy numbers from cells from HIV-infected individuals that were discontinuous and did not follow Gaussian distribution, Wilcoxon rank-sum tests were used to compare 2 groups (PMA/ionomycin vs. DMSO control) between paired clinical samples. In multiple groups of discontinuous variables with arbitrary detection limits, such as RTqPCR results and the frequency of HIV-1-infected cells, Friedman's nonparametric ANOVA test (2-tailed) with uncorrected Dunn's test was used to compare multiple groups (treatment vs. DMSO control). Western blot quantification followed Gaussian distribution and was tested by 1-way ANOVA with Fisher's least significant difference test. For the measurement of cellular canonical splicing, HIV-1-driven aberrant splicing, and HIV-1 RNA transcription, RNA reads came from the same RNA-Seq sample and were normalized by multiple-comparisons tests: repeated-measures 2-way ANOVA with the Geisser-Greenhouse correction and a post hoc analysis with Dunnett's multiple-comparisons test were used. All statistical calculations were done with GraphPad Prism v8.4. P values less than 0.05 were considered significant.

\section{Study approval}

The Yale University Institutional Review Board approved this study. All participants provided written consent. All HIV-1-infected individuals enrolled were on suppressive ART and maintained undetectable plasma HIV-1 RNA levels ( $<50$ copies $/ \mathrm{mL}$ ) for at least 6 months before enrollment. Characteristics of HIV-1-infected individuals are listed in Supplemental Table 2 .

\section{Author contributions}

YCH and YHJY conceptualized and designed the experiments. $\mathrm{YCH}$ developed the HIV-1-infected Jurkat T cell clones. YCH and KMJ performed the drug screen. KMJ and YHJY generated the dose- 
response curves. YHJY performed the in vitro and ex vivo studies and bioinformatic analysis. RMC, JC, RH, and SGD recruited the study participants. YCH, YHJY, and SGD wrote the manuscript.

\section{Acknowledgments}

We thank all study participants. We thank the NIH AIDS Reagent Program. This work was supported by a Yale Top Scholar award; a Rudolf J. Anderson Fellowship; NIH grants R01AI141009, R61DA047037, R21AI118402, and R37AI147868 (to YCH); a W.W. Smith AIDS Research Grant (to YCH); Johns Hopkins Center for AIDS Research (CFAR) Award P30AI094189 (to YCH); a
Gilead AIDS Research Grant (to YCH); a Gilead HIV Research Scholar Grant (to YCH); NIH BEAT-HIV Delaney Collaboratory UM1AI126620 (to YCH); and NIH CHEETAH P5OAI150464-13 (to $\mathrm{YCH}$ ). Additional support was provided by the UCSF/Gladstone Institute of Virology and Immunology CFAR (P30 AI027763), the CFAR Network of Integrated Systems (R24), and the Delaney AIDS Research Enterprise (AI096109 and A127966 to SGD).

Address correspondence to: Ya-Chi Ho, 295 Congress Ave, New Haven, Connecticut 06510, USA. Phone: 203.785.4052; Email: ya-chi.ho@yale.edu.
1. Finzi D, et al. Identification of a reservoir for HIV-1 in patients on highly active antiretroviral therapy. Science. 1997;278(5341):1295-1300.

2. Wong JK, et al. Recovery of replication-competent HIV despite prolonged suppression of plasma viremia. Science. 1997;278(5341):1291-1295.

3. Chun TW, et al. Presence of an inducible HIV-1 latent reservoir during highly active antiretroviral therapy. Proc Natl Acad Sci U S A. 1997;94(24):13193-13197.

4. De Scheerder MA, et al. HIV rebound is predominantly fueled by genetically identical viral expansions from diverse reservoirs. Cell Host Microbe. 2019;26(3):347-358.e7.

5. Archin NM, et al. Administration of vorinostat disrupts HIV-1 latency in patients on antiretroviral therapy. Nature. 2012;487(7408):482-485.

6. Korin YD, Brooks DG, Brown S, Korotzer A, Zack JA. Effects of prostratin on T-cell activation and human immunodeficiency virus latency. J Virol. 2002;76(16):8118-8123.

7. Nixon CC, et al. Systemic HIV and SIV latency reversal via non-canonical NF- $\kappa \mathrm{B}$ signalling in vivo. Nature. 2020;578(7793):160-165.

8. McBrien JB, et al. Robust and persistent reactivation of SIV and HIV by $\mathrm{N}-803$ and depletion of CD8(+) cells. Nature. 2020;578(7793):154-159.

9. Rasmussen TA, et al. Panobinostat, a histone deacetylase inhibitor, for latent-virus reactivation in HIV-infected patients on suppressive antiretroviral therapy: a phase $1 / 2$, single group, clinical trial. Lancet HIV. 2014;1(1):e13-e21.

10. Wei DG, et al. Histone deacetylase inhibitor romidepsin induces HIV expression in CD4 T cells from patients on suppressive antiretroviral therapy at concentrations achieved by clinical dosing. PLoS Pathog. 2014;10(4):e1004071.

11. Mota TM, et al. Integrated assessment of viral transcription, antigen presentation, and $\mathrm{CD}^{+} \mathrm{T}$ cell function reveal multiple limitations of class I selective HDACi during HIV-1 latency reversal. J Virol. 2020;94(9):e01845-19.

12. Boucau J, et al. The activation state of CD4 T cells alters cellular peptidase activities, HIV antigen processing, and MHC class I presentation in a sequence-dependent manner. JImmunol. 2019;202(10):2856-2872.

13. Böhnlein E, Lowenthal JW, Siekevitz M, Ballard DW, Franza BR, Greene WC. The same inducible nuclear proteins regulates mitogen activation of both the interleukin-2 receptor-alpha gene and type 1 HIV. Cell. 1988;53(5):827-836.

14. Jones KA, Peterlin BM. Control of RNA initiation and elongation at the HIV-1 promoter. Annu Rev Biochem. 1994;63:717-743.

15. Tyagi M, Pearson RJ, Karn J. Establishment of HIV latency in primary $\mathrm{CD}^{+}$cells is due to epigenetic transcriptional silencing and $\mathrm{P}-\mathrm{TEFb}$ restriction. J Virol. 2010;84(13):6425-6437.

16. Weinberger LS, Burnett JC, Toettcher JE, Arkin AP, Schaffer DV. Stochastic gene expression in a lentiviral positive-feedback loop: HIV-1 Tat fluctuations drive phenotypic diversity. Cell. 2005;122(2):169-182.

17. West MJ, Lowe AD, Karn J. Activation of human immunodeficiency virus transcription in $\mathrm{T}$ cells revisited: NF-kappaB p65 stimulates transcriptional elongation. J Virol. 2001;75(18):8524-8537.

18. Yukl SA, et al. HIV latency in isolated patient $\mathrm{CD}^{+} \mathrm{T}$ cells may be due to blocks in HIV transcriptional elongation, completion, and splicing. Sci Transl Med. 2018;10(430):eaap9927.

19. Coull JJ, et al. The human factors YY1 and LSF repress the human immunodeficiency virus type 1 long terminal repeat via recruitment of histone deacetylase 1. J Virol. 2000;74(15):6790-6799.

20. Kauder SE, Bosque A, Lindqvist A, Planelles $\mathrm{V}$, Verdin E. Epigenetic regulation of HIV-1 latency by cytosine methylation. PLoS Pathog. 2009;5(6):e1000495.

21. Van Lint C, Emiliani S, Ott M, Verdin E. Transcriptional activation and chromatin remodeling of the HIV-1 promoter in response to histone acetylation. ЕMBO J.1996;15(5):1112-1120.

22. Einkauf KB, et al. Intact HIV-1 proviruses accumulate at distinct chromosomal positions during prolonged antiretroviral therapy. J Clin Invest. 2019;129(3):988-998.

23. Roebuck KA, Gu DS, Kagnoff MF. Activating protein-1 cooperates with phorbol ester activation signals to increase HIV-1 expression. AIDS. 1996;10(8):819-826.

24. Kinoshita S, Su L, Amano M, Timmerman LA, Kaneshima H, Nolan GP. The T cell activation factor NF-ATc positively regulates HIV-1 replication and gene expression in T cells. Immunity. 1997;6(3):235-244.

25. Nabel G, Baltimore D. An inducible transcription factor activates expression of human immunodeficiency virus in T cells. Nature. 1987;326(6114):711-713.

26. Fennessey CM, et al. Genetically-barcoded SIV facilitates enumeration of rebound variants and estimation of reactivation rates in nonhuman primates following interruption of suppressive antiretroviral therapy. PLoS Pathog. 2017;13(5):e1006359.

27. Pinkevych M, et al. Correction: HIV reactivation from latency after treatment interruption occurs on average every 5-8 days-implications for HIV remission. PLoS Pathog. 2016;12(8):e1005745.

28. Pollack RA, et al. Defective HIV-1 proviruses are expressed and can be recognized by cytotoxic T lymphocytes, which shape the proviral landscape. Cell Host Microbe. 2017;21(4):494-506.e4.

29. Kearney MF, et al. Origin of rebound plasma HIV includes cells with identical proviruses that are transcriptionally active before stopping of antiretroviral therapy. J Virol. 2016;90(3):1369-1376.

30. Hunt PW, et al. T cell activation is associated with lower $\mathrm{CD}^{+}{ }^{+} \mathrm{T}$ cell gains in human immunodeficiency virus-infected patients with sustained viral suppression during antiretroviral therapy. JInfect Dis. 2003;187(10):1534-1543.

31. Hunt PW, et al. Relationship between T cell activation and $\mathrm{CD} 4^{+} \mathrm{T}$ cell count in HIV-seropositive individuals with undetectable plasma HIV RNA levels in the absence of therapy. J Infect Dis. 2008;197(1):126-133.

32. Akiyama H, Miller CM, Ettinger CR, Belkina AC, Snyder-Cappione JE, Gummuluru S. HIV-1 intron-containing RNA expression induces innate immune activation and $\mathrm{T}$ cell dysfunction. Nat Commun. 2018;9(1):3450.

33. McCauley SM, et al. Intron-containing RNA from the HIV-1 provirus activates type I interferon and inflammatory cytokines. Nat Commun. 2018;9(1):5305.

34. Hatano $\mathrm{H}$, et al. Cell-based measures of viral persistence are associated with immune activation and programmed cell death protein 1 (PD-1)-expressing CD $4^{+}$T cells. J Infect Dis. 2013;208(1):50-56.

35. Lederman MM, Funderburg NT, Sekaly RP, Klatt NR, Hunt PW. Residual immune dysregulation syndrome in treated HIV infection. Adv Immunol. 2013;119:51-83.

36. Hunt PW, Lee SA, Siedner MJ. Immunologic biomarkers, morbidity, and mortality in treated HIV infection. J Infect Dis. 2016;214(suppl 2):S44-S50.

37. Hsue PY, et al. Progression of atherosclerosis as assessed by carotid intima-media thickness in patients with HIV infection. Circulation. 2004;109(13):1603-1608.

38. Hsue PY, Deeks SG, Hunt PW. Immunologic basis of cardiovascular disease in HIV-infected adults. J Infect Dis. 2012;205(suppl 3):S375-S382.

39. Deeks SG. Immune dysfunction, inflammation, and accelerated aging in patients on antiretrovi- 
ral therapy. Top HIV Med. 2009;17(4):118-123.

40. Hodes RJ, et al. Disease drivers of aging. Ann N Y Acad Sci. 2016;1386(1):45-68.

41. Wang Y, et al. HIV-1-induced cytokines deplete homeostatic innate lymphoid cells expand TCF7-dependent memory NK cells. Nature Immunology. 2020;21(3):274-286.

42. Yang HC, et al. Small-molecule screening using a human primary cell model of HIV latency identifies compounds that reverse latency without cellular activation. JClin Invest. 2009;119(11):3473-3486.

43. Yang HC, Shen L, Siliciano RF, Pomerantz JL. Isolation of a cellular factor that can reactivate latent HIV-1 without T cell activation. Proc Natl Acad Sci U S A. 2009;106(15):6321-6326.

44. Zhu J, et al. Reactivation of latent HIV-1 by inhibition of BRD4. Cell Rep. 2012;2(4):807-816.

45. Hayashi T, Jean M, Huang H, Simpson S, Santoso NG, Zhu J. Screening of an FDA-approved compound library identifies levosimendan as a novel anti-HIV-1 agent that inhibits viral transcription. Antiviral Res. 2017;146:76-85.

46. Laird GM, Eisele EE, Rabi SA, Nikolaeva D, Siliciano RF. A novel cell-based high-throughput screen for inhibitors of HIV-1 gene expression and budding identifies the cardiac glycosides. JAntimicrob Chemother. 2014;69(4):988-994.

47. Mousseau G, Valente S. Strategies to block HIV transcription: focus on small molecule Tat inhibitors. Biology (Basel). 2012;1(3):668-697.

48. Besnard E, et al. The mTOR complex controls HIV latency. Cell Host Microbe. 2016;20(6):785-797.

49. Das B, et al. Estrogen receptor-1 is a key regulator of HIV-1 latency that imparts gender-specific restrictions on the latent reservoir. Proc Natl Acad Sci U S A. 2018;115(33):E7795-E7804.

50. Lacombe B, Morel M, Margottin-Goguet F, Ramirez BC. Specific inhibition of HIV infection by the action of spironolactone in T cells. J Virol. 2016;90(23):10972-10980.

51. Chapuis AG, et al. Effects of mycophenolic acid on human immunodeficiency virus infection in vitro and in vivo. Nat Med. 2000;6(7):762-768.

52. Gavegnano C, et al. Novel mechanisms to inhibit HIV reservoir seeding using Jak inhibitors. PLoS Pathog. 2017;13(12):e1006740.

53. Genovese MC, et al. Effect of filgotinib vs placebo on clinical response in patients with moderate to severe rheumatoid arthritis refractory to disease-modifying antirheumatic drug therapy: the FINCH 2 randomized clinical trial. JAMA. 2019;322(4):315-325.

54. van der Heijde D, et al. Efficacy and safety of filgotinib, a selective Janus kinase 1 inhibitor, in patients with active ankylosing spondylitis (TORTUGA): results from a randomised, placebo-controlled, phase 2 trial. Lancet. 2018;392(10162):2378-2387.

55. Mease P, et al. Efficacy and safety of filgotinib, a selective Janus kinase 1 inhibitor, in patients with active psoriatic arthritis (EQUATOR): results from a randomised, placebo-controlled, phase 2 trial. Lancet. 2018;392(10162):2367-2377.

56. Vermeire $\mathrm{S}$, et al. Clinical remission in patients with moderate-to-severe Crohn's disease treated with filgotinib (the FITZROY study): results from a phase 2, double-blind, ran- domised, placebo-controlled trial. Lancet. 2017;389(10066):266-275.

57. Schröder AR, Shinn P, Chen H, Berry C, Ecker JR, Bushman F. HIV-1 integration in the human genome favors active genes and local hotspots. Cell. 2002;110(4):521-529.

58. Han $\mathrm{Y}$, et al. Resting $\mathrm{CD} 4^{+} \mathrm{T}$ cells from human immunodeficiency virus type 1 (HIV-1)-infected individuals carry integrated HIV-1 genomes within actively transcribed host genes. J Virol. 2004;78(12):6122-6133.

59. Gavegnano C, Detorio M, Montero C, Bosque A, Planelles V, Schinazi RF. Ruxolitinib and tofacitinib are potent and selective inhibitors of HIV-1 replication and virus reactivation in vitro. Antimicrob Agents Chemother. 2014;58(4):1977-1986.

60. Chao SH, et al. Flavopiridol inhibits P-TEFb and blocks HIV-1 replication. J Biol Chem. 2000;275(37):28345-28348.

61. Martin AR, Pollack RA, Capoferri A, Ambinder RF, Durand CM, Siliciano RF. Rapamycin-mediated mTOR inhibition uncouples HIV-1 latency reversal from cytokine-associated toxicity. J Clin Invest. 2017;127(2):651-656.

62. Contreras X, Barboric M, Lenasi T, Peterlin BM. HMBA releases P-TEFb from HEXIM1 and 7SK snRNA via PI3K/Akt and activates HIV transcription. PLoS Pathog. 2007;3(10):1459-1469.

63. Daelemans D, et al. A synthetic HIV-1 Rev inhibitor interfering with the CRM1-mediated nuclear export. Proc Natl Acad Sci U S A. 2002;99(22):14440-14445.

64. Reeves DB, Duke ER, Hughes SM, Prlic M, Hladik F, Schiffer JT. Anti-proliferative therapy for HIV cure: a compound interest approach. Sci Rep. 2017;7(1):4011.

65. Vanhoutte F, et al. Efficacy, safety, pharmacokinetics, and pharmacodynamics of filgotinib, a selective JAK-1 inhibitor, after short-term treatment of rheumatoid arthritis: results of two randomized phase IIa trials. Arthritis Rheumatol. 2017;69(10):1949-1959.

66. Karim A, Zagarella J, Hutsell TC, Chao A, Baltes BJ. Spironolactone. II. Bioavailability. Clin Pharmacol Ther. 1976;19(2):170-176.

67. Bullen CK, Laird GM, Durand CM, Siliciano JD, Siliciano RF. New ex vivo approaches distinguish effective and ineffective single agents for reversing HIV-1 latency in vivo. Nat Med. 2014;20(4):425-429.

68. Shan L, et al. A novel PCR assay for quantification of HIV-1 RNA. J Virol. 2013;87(11):6521-6525.

69. Palmer $S$, et al. New real-time reverse transcriptase-initiated PCR assay with single-copy sensitivity for human immunodeficiency virus type 1 RNA in plasma. J Clin Microbiol. 2003;41(10):4531-4536

70. Massanella M, Gianella S, Lada SM, Richman DD, Strain MC. Quantification of total and 2-LTR (long terminal repeat) HIV DNA, HIV RNA and herpesvirus DNA in PBMCs. Bio Protoc. 2015;5(11):e1492.

71. Liu R, et al. Single-cell transcriptional landscapes reveal HIV-1-driven aberrant host gene transcription as a potential therapeutic target. Science Transl Med.2020;12(543):eaaz0802.

72. Maldarelli F, et al. HIV latency. Specific HIV integration sites are linked to clonal expan- sion and persistence of infected cells. Science. 2014;345(6193):179-183.

73. Nobles CL, et al. CD19-targeting CAR T cell immunotherapy outcomes correlate with genomic modification by vector integration. JClin Invest. 2020;130(2):673-685

74. Katzav S, Cleveland JL, Heslop HE, Pulido D. Loss of the amino-terminal helix-loop-helix domain of the vav proto-oncogene activates its transforming potential. Mol Cell Biol. 1991;11(4):1912-1920.

75. Krämer A, Green J, Pollard J, Tugendreich S. Causal analysis approaches in Ingenuity Pathway Analysis. Bioinformatics. 2014;30(4):523-530.

76. Subramanian A, et al. Gene set enrichment analysis: a knowledge-based approach for interpreting genome-wide expression profiles. Proc Natl Acad Sci U S A. 2005;102(43):15545-15550.

77. Middleton R, et al. IRFinder: assessing the impact of intron retention on mammalian gene expression. Genome Biol. 2017;18(1):51.

78. Liberzon A, Subramanian A, Pinchback R, Thorvaldsdóttir $\mathrm{H}$, Tamayo P, Mesirov JP. Molecular signatures database (MSigDB) 3.0. Bioinformatics. 2011;27(12):1739-1740.

79. Shannon P, et al. Cytoscape: a software environment for integrated models of biomolecular interaction networks. Genome Res 2003;13(11):2498-2504.

80. Wong RW, Balachandran A, Ostrowski MA, Cochrane A. Digoxin suppresses HIV-1 replication by altering viral RNA processing. PLoS Pathog. 2013;9(3):e1003241

81. Wong R, Balachandran A, Mao AY, Dobson W, Gray-Owen S, Cochrane A. Differential effect of CLK SR Kinases on HIV-1 gene expression: potential novel targets for therapy. Retrovirology. 2011;8:47.

82. Serquiña AK, Das SR, Popova E, Ojelabi OA, Roy CK, Göttlinger HG. UPF1 is crucial for the infectivity of human immunodeficiency virus type 1 progeny virions. J Virol. 2013;87(16):8853-8861.

83. Ajamian L, et al. Unexpected roles for UPF1 in HIV-1 RNA metabolism and translation. RNA. 2008;14(5):914-927.

84. Pak V, Eifler TT, Jager S, Krogan NJ, Fujinaga K, Peterlin BM. CDK11 in TREX/THOC regulates HIV mRNA 3' end processing. Cell Host Microbe. 2015;18(5):560-570.

85. Yoh SM, Lucas JS, Jones KA. The Iws1:Spt6:CTD complex controls cotranscriptional mRNA biosynthesis and HYPB/Setd2-mediated histone H3K36 methylation. Genes Dev. 2008;22(24):3422-3434.

86. Lichinchi $G$, et al. Dynamics of the human and viral m(6)A RNA methylomes during HIV-1 infection of T cells. Nat Microbiol. 2016;1:16011.

87. Lv L, et al. Vpr targets TET2 for degradation by CRL4(VprBP) E3 ligase to sustain IL-6 expression and enhance HIV-1 replication. Mol Cell. 2018;70(5):961-70.e5.

88. Sorin M, et al. Recruitment of a SAP18HDAC1 complex into HIV-1 virions and its requirement for viral replication. PLoS Pathog. 2009;5(6):e1000463.

89. Margolis DM. Histone deacetylase inhibitors and HIV latency. Curr Opin HIV AIDS. 2011;6(1):25-29. 90. Barton KM, et al. Selective HDAC inhibition for 


\section{RESEARCH ARTICLE}

the disruption of latent HIV-1 infection. PLoS One. 2014;9(8):e102684.

91. Pauls E, et al. Cell cycle control and HIV-1 susceptibility are linked by CDK6-dependent CDK2 phosphorylation of SAMHD1 in myeloid and lymphoid cells. JImmunol. 2014;193(4):1988-1997.

92. Ammosova T, et al. Phosphorylation of HIV-1 Tat by CDK2 in HIV-1 transcription. Retrovirology. 2006;3:78.

93. Boehm D, et al. SMYD2-mediated histone methylation contributes to HIV-1 latency. Cell Host Microbe. 2017;21(5):569-579.e6.

94. Guendel I, et al. BRCA1 functions as a novel transcriptional cofactor in HIV-1 infection. Virol J. 2015;12:40.

95. Keedy KS, Archin NM, Gates AT, Espeseth A, Hazuda DJ, Margolis DM. A limited group of class I histone deacetylases acts to repress human immunodeficiency virus type 1 expression. J Virol. 2009;83(10):4749-4756.

96. Hao S, Baltimore D. RNA splicing regulates the temporal order of TNF-induced gene expression. Proc Natl Acad Sci U SA. 2013;110(29):11934-11939.

97. $\mathrm{Ni} \mathrm{T}$, et al. Global intron retention mediated gene regulation during $\mathrm{CD} 4^{+} \mathrm{T}$ cell activation. Nucleic Acids Res. 2016;44(14):6817-6829.

98. Braunschweig U, et al. Widespread intron retention in mammals functionally tunes transcriptomes. Genome Res. 2014;24(11):1774-1786.

99. Frankiw L, et al. BUD13 promotes a type I interferon response by countering intron retention in
Irf7. Mol Cell. 2019;73(4):803-814.e6.

100.Sherrill-Mix S, Ocwieja KE, Bushman FD. Gene activity in primary $\mathrm{T}$ cells infected with HIV89.6: intron retention and induction of genomic repeats. Retrovirology. 2015;12:79.

101. Kutluay SB, et al. Genome-wide analysis of heterogeneous nuclear ribonucleoprotein (hnRNP) binding to HIV-1 RNA reveals a key role for hnRNP H1 in alternative viral mRNA splicing. JVirol. 2019;93(21):e01048-19.

102. Stavrou S, Aguilera AN, Blouch K, Ross SR. DDX41 recognizes RNA/DNA retroviral reverse transcripts and is critical for in vivo control of murine leukemia virus infection. mBio. 2018;9(3):e00923-18.

103. Massanella M, et al. Improved assays to measure and characterize the inducible HIV reservoir. EBioMedicine. 2018;36:113-121.

104. Rosenbloom DI, Elliott O, Hill AL, Henrich TJ, Siliciano JM, Siliciano RF. Designing and interpreting limiting dilution assays: general principles and applications to the latent reservoir for human immunodeficiency virus-1. Open Forum Infect Dis. 2015;2(4):ofv123.

105.Zhyvoloup A, et al. Digoxin reveals a functional connection between HIV-1 integration preference and T-cell activation. PLoS Pathog. 2017;13(7):e1006460.

106.Dvir A, Conaway RC, Conaway JW. A role for TFIIH in controlling the activity of early RNA polymerase II elongation complexes. Proc Natl
Acad Sci U S A. 1997;94(17):9006-9010.

107. Mondal N, Parvin JD. DNA topoisomerase IIalpha is required for RNA polymerase II transcription on chromatin templates. Nature. 2001;413(6854):435-438.

108. Pinzone MR, et al. Longitudinal HIV sequencing reveals reservoir expression leading to decay which is obscured by clonal expansion. Nat Commun. 2019;10(1):728.

109. Purcell DF, Martin MA. Alternative splicing of human immunodeficiency virus type $1 \mathrm{mRNA}$ modulates viral protein expression, replication, and infectivity. JVirol. 1993;67(11):6365-6378.

110.Dlamini Z, Hull R. Can the HIV-1 splicing machinery be targeted for drug discovery? HIV AIDS (Auckl). 2017;9:63-75.

111. Pinzone MR, et al. Longitudinal HIV sequencing reveals reservoir expression leading to decay which is obscured by clonal expansion. Nat Commun. 2019;10(1):728.

112. Cesana D, et al. HIV-1-mediated insertional activation of STAT5B and BACH2 trigger viral reservoir in T regulatory cells. Nat Commun. 2017;8(1):498.

113. Ho YC, et al. Replication-competent noninduced proviruses in the latent reservoir increase barrier to HIV-1 cure. Cell. 2013;155(3):540-551.

114. Merico D, Isserlin R, Stueker O, Emili A, Bader GD. Enrichment map: a network-based method for gene-set enrichment visualization and interpretation. PLoS One. 2010;5(11):e13984. 